\title{
Hamiltonian Representation of Higher Order Partial Differential Equations with Boundary Energy Flows
}

\author{
Gou Nishida \\ Department of Mechanical and Environmental Informatics, Graduate School of Information Science and \\ Engineering, Tokyo Institute of Technology, Tokyo, Japan \\ Email: g.nishida@ieee.org
}

Received 27 September 2015; accepted 24 November 2015; published 27 November 2015

Copyright (C 2015 by author and Scientific Research Publishing Inc.

This work is licensed under the Creative Commons Attribution International License (CC BY). http://creativecommons.org/licenses/by/4.0/

(c) (7) Open Access

\begin{abstract}
This paper presents a system representation that can be applied to the description of the interaction between systems connected through common boundaries. The systems consist of partial differential equations that are first order with respect to time, but spatially higher order. The representation is derived from the instantaneous multisymplectic Hamiltonian formalism; therefore, it possesses the physical consistency with respect to energy. In the interconnection, particular pairs of control inputs and observing outputs, called port variables, defined on the boundaries are used. The port variables are systematically introduced from the representation.
\end{abstract}

\section{Keywords}

Symplectic Structure, Dirac Structure, Hamiltonian Systems, Passivity, Partial Differential Equations, Nonlinear Systems

\section{Introduction}

Energy is one of the most important concepts for describing physical systems. In analytical mechanics, an energy in the systems can be interpreted as a Hamiltonian. Hamiltonian systems can be characterized by symplectic structures [1] derived from the skew symmetry that cotangent bundles possess. Hamiltonian systems and their symplectic structures have been widely applied not only in physics, but in engineering, particularly, control theory [2] [3]. Specifically, in an electrical circuit, an energy is defined as the time integral of the product of currents and voltages. Energy flows between each circuit also balance if there is no dissipative element. Furthermore, the sum of currents balances between inflows and outflows at any node, and the directed sum of vol- 
tages around any closed loop is zero according to Kirchhoff law. Indeed, such properties have been generalized to various physical systems in terms of port-Hamiltonian systems [2] originated in bondgraph theory [4]. The above particular pairs with the physical dimension of power are called port variables, and the energy balances can be regarded as passivity [2]. A system is passive if and only if a finite amount of energy can be extracted from the system. In other words, energy changes in interactions can be observed by the port variables, and a supplied energy is less than a stored energy if a system is passive. Passivity-based controls via port-Hamiltonian system representations have been frequently used in control designs [3].

This paper proposes the port-Hamiltonian representation of systems of higher order partial differential equations defined on a domain with a boundary. The representation can be formally formulated from the viewpoint of the multisymplectic formalism [5] [6] under the assumption of first order with respect to time, but possibly higher order with respect to spatial variables. The port representation for Hamiltonian systems with boundary energy flows was initiated by the distributed port-Hamiltonian system in [7]. The systems satisfy a power balance defined on the boundary; therefore, it can describe the interaction between the systems connected through common boundaries. Thus, passivity-based controls in this formulation can be enhanced as boundary energy controls. Various aspects of the distributed port-Hamiltonian systems have been studied, e.g., the implicit representation of distributed port-Hamiltonian systems [8], and the relationship between field equations and distributed port-Hamiltonian systems [9]-[11]. The higher order representation of the distributed port-Hamiltonian systems has been proposed in, e.g., [12] [13]; however, they are not related with the multisymplectic formalism.

Thus, we first relate higher order partial differential equations with the implicit Hamiltonian systems [14]. Next, we describe the implicit representation as a Dirac structure defined over the multisymplectic manifold in analogy with the first order formalizations [15]-[17]. Dirac structures [18] [19] are a unified concept of symplectic and Poisson structures. Then, we derive the Stokes variational differential from the fact that higher order derivatives yield variations of boundary port variables through integration by parts and Stokes theorem. Finally, we shows that the boundary energy balance and the Stokes-Dirac structure [7] [20] that is an extended Dirac structure for distributed port-Hamiltonian systems can be defined in the proposed higher order field port Hamiltonian systems with boundary energy flows.

This paper is organized as follows: In Section 2, we make a brief summary of port-Hamiltonian systems and explain the motivation of this study. Section 3 introduces mathematical preliminaries from some references. Section 4 presents the following three concepts under the assumption of time-spatial splitting: 1) an implicit Hamiltonian representation using the dual structure derived from the multisymplectic instantaneous formalism, 2) Stokes variational differential derived from the integration by parts formula, and 3) the implicit higher order field port Hamiltonian representation with boundary. Section 5 introduces the formal port representation for higher order partial differential equations from the implicit Hamiltonian representation. We call it higher order field port Hamiltonian systems with boundary energy flows. Finally, Section 6 illustrates two modeling examples.

\section{Summary of Port-Hamiltonian Representations}

This section explains the concept of port-Hamiltonian systems by means of a simple example of coupled multiphysical models, and the motivation of this work.

\subsection{Port Representation for Lumped Parameter Energy Conserving Physical Systems}

Let us consider the following model of the direct current motor consisting of an electrical circuit and an armature:

$$
L \frac{\mathrm{d} i}{\mathrm{~d} t}+R i=u-K \omega, \quad J \frac{\mathrm{d} \omega}{\mathrm{d} t}+B \omega=N i,
$$

where $i=i(t)=\mathrm{d} q / \mathrm{d} t$ is the current that is the time derivative of the electric charge $q=q(t)$, $\omega=\omega(t)=\mathrm{d} \theta / \mathrm{d} t$ is the velocity of the angle $\theta=\theta(t)$, and $u$ is the input voltage. In (1), the following constants are defined: the inductance $L$, the resistance $R$, the back electromotive force constant $K$, the inertia moment $J$, the viscous friction constant $B$, and the torque constant $N$. When the dissipative elements and the input are null, i.e., $R=B=0$ and $u=0$, the system (1) is energy conserving, and it can be formulated as the following standard Hamiltonian system: 


$$
\dot{p}_{q}^{t}=-\frac{\partial \mathcal{H}}{\partial q}, \quad \dot{q}=\frac{\partial \mathcal{H}}{\partial p_{q}^{t}}, \quad \dot{p}_{\theta}^{t}=-\frac{\partial \mathcal{H}}{\partial \theta}, \quad \dot{\theta}=\frac{\partial \mathcal{H}}{\partial p_{\theta}^{t}},
$$

where we have defined the Hamiltonian and the momenta as follows:

$$
\begin{gathered}
\mathcal{H}=\dot{q} p_{q}^{t}+\dot{\theta} p_{\theta}^{t}-\mathcal{L}, \quad \mathcal{L}=\frac{1}{2} L \dot{q}^{2}+\frac{1}{2} J \dot{\theta}^{2}-\kappa_{1} \dot{\theta} q+\kappa_{2} \theta \dot{q}, \\
p_{q}^{t}=\frac{\partial \mathcal{L}}{\partial \dot{q}}=L \dot{q}+\kappa_{2} \theta, \quad p_{\theta}^{t}=\frac{\partial \mathcal{L}}{\partial \dot{\theta}}=J \dot{\theta}-\kappa_{1} q
\end{gathered}
$$

with $K:=\kappa_{2}-\kappa_{1}$ and $N:=\kappa_{1}+\kappa_{2}$ for certain $\kappa_{1}$ and $\kappa_{2}$.

We shall augment the Hamiltonian system (2) as the following port-Hamiltonian system with the dissipations $-R \dot{q}$ and $-B \dot{\theta}$ and the input $u$ :

$$
\left\{\begin{array}{l}
{\left[\begin{array}{l}
f_{1}^{p} \\
f_{1}^{q}
\end{array}\right]=\left[\begin{array}{cc}
-R & -1 \\
1 & 0
\end{array}\right]\left[\begin{array}{l}
e_{1}^{p} \\
e_{1}^{q}
\end{array}\right]+\left[\begin{array}{l}
1 \\
0
\end{array}\right] u, \quad y^{q}=\left[\begin{array}{ll}
1 & 0
\end{array}\right]\left[\begin{array}{l}
e_{1}^{p} \\
e_{1}^{q}
\end{array}\right],} \\
{\left[\begin{array}{l}
f_{2}^{p} \\
f_{2}^{q}
\end{array}\right]=\left[\begin{array}{cc}
-B & -1 \\
1 & 0
\end{array}\right]\left[\begin{array}{l}
e_{2}^{p} \\
e_{2}^{q}
\end{array}\right],}
\end{array}\right.
$$

where we have defined the following variables called port variables:

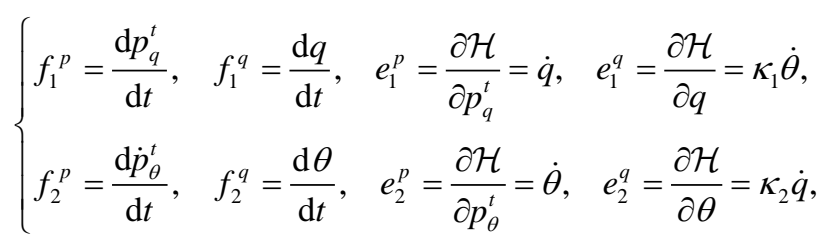

and, in particular, $f_{j}^{i}$ and $e_{j}^{i}$ for $i=\{p, q\}$ and $j=\{1,2\}$ are called flows and efforts, respectively. Here, we can see that the electrical and mechanical subsystems are coupled by the interconnection of the effort variables: $\dot{q}$ and $\dot{\theta}$.

Then, the summation consisting of the products of the pairs of the port variables is equivalent to the time derivative of the Hamiltonian, i.e., the total energy change of the system. Indeed, we can directly calculate the following power balance:

$$
\frac{\mathrm{d} \mathcal{H}}{\mathrm{d} t}=f_{1}^{p} e_{1}^{p}+f_{1}^{q} e_{1}^{q}+f_{2}^{p} e_{2}^{p}+f_{2}^{q} e_{2}^{q}=-R \dot{q}^{2}-B \dot{\theta}^{2}+u y^{q},
$$

where we have used the relation $y^{q}=\dot{q}=e_{1}^{p}$. The relation (7) means that the dissipations $-R \dot{q}^{2}$ and $-B \dot{\theta}^{2}$ stabilize the system by decreasing the energy, and the product $u y^{q}$ of the input and the output may affects the dissipation rate. Furthermore, the Hamiltonian can be controlled if we can find a suitable input satisfying $\tilde{\mathcal{H}}\left(t_{1}\right)-\tilde{\mathcal{H}}\left(t_{0}\right)=\int_{t_{0}}^{t_{1}} u y^{q} \mathrm{~d} t$ for the desired Hamiltonian $(\mathcal{H}+\tilde{\mathcal{H}})$ in the time interval $\left[t_{0}, t_{1}\right]$. A finite amount of energy changes in interactions can be precisely observed by the product of an input-output pair if the system is passive. Hence, these controls are called passivity-based controls.

\subsection{Distributed Port-Hamiltonian Systems}

In the case of distributed parameter systems, the representation is called a distributed port-Hamiltonian system, and has the following formal structure:

$$
\left[\begin{array}{l}
f^{p} \\
f^{q}
\end{array}\right]=\left[\begin{array}{cc}
0 & (-1)^{r} \mathrm{~d} \\
\mathrm{~d} & 0
\end{array}\right]\left[\begin{array}{l}
e^{p} \\
e^{q}
\end{array}\right], \quad\left[\begin{array}{l}
f^{b} \\
e^{b}
\end{array}\right]=\left[\begin{array}{c}
\left.e^{p}\right|_{\partial \mathcal{S}} \\
\left.(-1)^{p} e^{q}\right|_{\partial \mathcal{S}}
\end{array}\right]
$$

called the Stokes-Dirac structure defined on the system domain $\mathcal{S}$ with the boundary $\partial \mathcal{S}$ (see [7] for details), where $d$ is the exterior differential operator, we have defined 


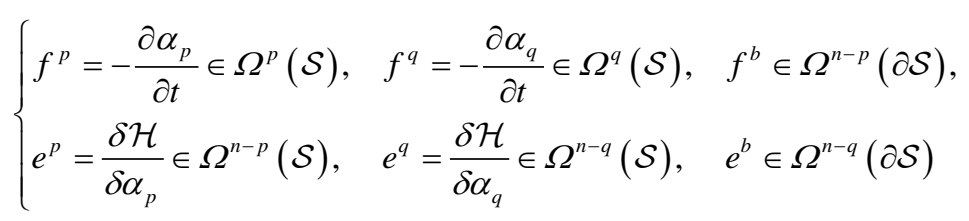

for a Hamiltonian functional $\mathscr{H}=\int_{\mathcal{S}} \mathcal{H}, \Omega^{k}$ is the space of differential $k$-forms, $\delta / \delta \alpha_{i}$ is the variational derivative with respect to the differential form $\alpha_{i}, r=p q+1$, and $p+q=n+1$. Then, the power balance (7) is extended to the following relation described by differential forms:

$$
\frac{\mathrm{d} \mathscr{K}}{\mathrm{d} t}=\int_{\mathcal{S}}\left(f_{p} \wedge e_{p}+f_{q} \wedge e_{q}\right)=\int_{\partial \mathcal{S}} e_{p} \wedge e_{q} .
$$

The boundary integral term in (10) is generated from the domain integral term by Stokes theorem: $\int_{\mathcal{S}} \mathrm{d} \omega=\int_{\partial \mathcal{S}} \omega$ for an $n$-form $\mathrm{d} \omega$ in the $n$-dimensional domain $\mathcal{S}$. Hence, the passivity-based controls can be enhanced as boundary energy controls by regarding $e_{p}$ and $e_{q}$ as a input-output pair, i.e., boundary port variables.

\subsection{Motivation}

As we have seen above, the port-Hamiltonian representations are important for the control of, e.g., nonlinear systems, distributed parameter systems, higher order systems and multi-physical systems from which it is difficult to obtain analytical solutions in a closed form. This paper derives a formal port-Hamiltonian representation of a given partial differential equation including higher order derivatives in terms of the multisymplectic formalism.

In this paper, we assume that a given system of partial differential equations is determined by variational problems. Such a system must be regarded as an energy conserving physical system [15] through Legendre transformations that map Lagrangian systems to Hamiltonian systems. This assumption comes from the fact that any system can be decomposed into a variational subsystem that can be determined by variational calculus and a non-variational subsystem that cannot be introduced from any Lagrangian on a contractible manifold [21]. For example, as we seen in (1), the dissipative terms $-R \dot{q}$ and $-B \dot{\theta}$ cannot be derived from any Lagrangian or Hamiltonian. On the other hand, Lagrangians of the first subsystem can be explicitly calculated by homotopy operators in terms of the exactness of vertical differential forms in variational bi-complex [22] [23]. Hence, we consider only the variational subsystems in this study.

\section{Mathematical Preliminary}

Mathematical notations used in this paper basically conform to those of the references [22] [24] [25].

\subsection{Multi index for Higher Order Derivatives}

Let $X$ be an $(m+1)$-dimensional manifold. Let $Q$ be a fiber manifold on $X$, and consider the $r$-th order jet bundle $J^{r} Q$ over $Q$. We denote the local coordinates of $X, Q$, and $J^{r} Q$, respectively, by $x^{i},\left(x^{i}, q^{a}\right)$, and $\left(x^{i}, q_{I}^{a}\right)$, where $0 \leq i \leq m$, and $1 \leq a \leq l$. The multi index $I$ describes all variables of the repeated combination of $x^{i}$ that mean higher order derivatives with respect to the variable, e.g., $q_{x^{i} x^{j}}^{a}=\partial^{2} q^{a} / \partial x^{i} \partial x^{j}$. We the order of $I$ by $|I|$, and it is used as $0 \leq|I| \leq r$ that means all derivatives up to the $r$-th order. Let $x^{0}$ be the time coordinate for $t \in \mathbb{R}$ and let $x^{s}$ be the spatial coordinates for $1 \leq s \leq m$. In some case, we use the abbreviation such as $q_{t}^{a}=q_{x^{0}}^{a}=q_{0}^{a}$.

Example 1. Let $m=1, l=1$, and $r=2$. We define the local coordinates of $X$ by $\left(x^{0}, x^{1}\right)=(t, y)$, and those of $Q$ by $\left(x^{0}, x^{1} ; q^{1}\right)=(t, y ; q)$. Then, the local coordinates of the jet bundle $J^{2} Q$ are $\left(x^{0}, x^{1} ; q_{I}^{1}\right)=$ $\left(t, y ; q, q_{t}, q_{y}, q_{t t}, q_{t y}, q_{y y}\right)$ for all $0 \leq|I| \leq 2$. Note that $q$ is described as a function of $t, y$; however, each element of $q_{I}$ is regarded as independent variables on the bundle. By using the summation convention, for example, we can interpret such as $\left(\partial \mathcal{L} / \partial q_{I}\right) \mathrm{d} q_{I}=\left(\partial \mathcal{L} / \partial q_{t}\right) \mathrm{d} q_{t}+\left(\partial \mathcal{L} / \partial q_{y}\right) \mathrm{d} q_{y}$ for $|I|=1$. 


\subsection{Time-Spatial Splitting of State Space}

Variational problems can be formalized as follows.

Definition 1. Consider the $(m+1,0)$-form $\mathcal{L}:=\mathcal{L} \mathrm{d} x \in \Omega^{m+1,0}\left(J^{r} Q\right)$ as a Lagrangian density of a functional $\int_{\mathcal{Y}} \mathcal{L} \mathrm{d} x$. We define the variational derivative $\mathrm{d} \mathcal{L}$ of the Lagrangian density as the $(m+1,1)$-form

$$
\mathrm{d}_{v} \mathcal{L} \mathrm{d} x=\frac{\partial \mathcal{L}}{\partial q_{I}^{a}} \mathrm{~d} q_{I}^{a} \wedge \mathrm{d} x \in \Omega^{m+1,1}\left(J^{r} Q\right)
$$

that determines the stationary condition $\mathrm{d}_{v} \mathcal{L} \equiv 0$ of the variational problem, where $0 \leq|I| \leq r$, and $\mathrm{d}_{v}$ is the vertical differential operator (see Sections B and C). In the following discussions, the variational derivative $\mathrm{d} \mathcal{L}$ is lifted on $J^{2 r-1} Q$.

Let us consider the following control system as a main objective.

Assumption 1. A given system is defined on a contractible domain $\mathcal{S}$ with a boundary $\partial \mathcal{S}$, and it can be derived from a Lagrangian density in functional forms including derivatives that are first order with respect to the time coordinate and up to $(r-1)$-th order with respect to spatial coordinates.

Under this assumption, the multi index $I$ up to $r$-th order used for describing $r$-th order Lagrangians can be defined as $I=\{K, K t\}$, where the multi index $K$ is of spatial coordinates for $0 \leq|K| \leq r-1$.

Example 2. In the case of $m=2, l=1$ and $r=2$, let $\left(x^{0}, x^{1}, x^{2} ; q^{1}\right)=(t, y, z ; q)$ be a local coordinates of $Q$ on $X$. Then, by defining $K=\{\varnothing, y, z\}$ and $I=\{K, K t\}=\{\varnothing, y, z, t, y t, z t\}$, the local coordinates of $J^{2} Q$ can be described as $\left(x^{0}, x^{1}, x^{2} ; q_{I}^{1}\right)=\left(t, y, z ; q_{I}\right)$. Without Assumption 1 , I may include $t t, y y, z z$.

Furthermore, the following second assumption is important when we use the multisymplectic instantaneous formalism (see Section E).

Definition 2. Let us consider the time-spatial split domain $\mathcal{Y}=\mathcal{T} \times \mathcal{S} \subset \mathbb{R} \times \Sigma$ consisting of a time interval $\mathcal{T} \subset \mathbb{R}$ and a spatial domain $\mathcal{S} \subset \Sigma$. Then, a system defined on $\mathcal{Y}$ at an instantaneous time $t \in \mathcal{T}$ is defined on $\mathcal{S}$.

Assumption 2. A Lagrangian density $\mathcal{L} \in \Omega^{m+1,0}\left(J^{r} Q\right)$ restricted to $\mathcal{S}$ can be described as $\left.\mathcal{L} \mathrm{d} x^{s} \in \Omega^{m, 0}\left(J^{r} Q\right)\right|_{\mathcal{S}}$ at a time $t \in \mathcal{T}$ in $\mathcal{Y}=\mathcal{T} \times \mathcal{S}$, where we denote the spatial (horizontal) volume form $\mathrm{d} x^{1} \wedge \cdots \wedge \mathrm{d} x^{m}$ by $\mathrm{d} x^{s}$.

Note that, we treat variables including time derivatives, e.g., $q_{K t}^{a}$ in a bundle restricted to the spatial domain $\mathcal{S}$ in this setting.

\section{Implicit Hamiltonian Representation Induced from Distributions}

In this section, we present a symplectic structure for distributions determined by partial differential equations in terms of the implicit Hamiltonian representation [14]. A distribution is a subbundle of a tangent bundle that is defined by a system dynamics, external constraints, and internal constraints generated by degenerate Lagrangians. On the other hand, a field Hamiltonian system is defined by the covariant Hamiltonian in the multisymplectic formalism [6]. However, the covariant Hamiltonian does not correspond to the typical Hamiltonian that are constant with respect to time evolution, e.g., for particle systems, but the instantaneous Hamiltonian derived from the time-spatial splitting.

\subsection{Distributions}

Definition 3. Consider a system of Pfaff equations $\mathcal{P}=\left\{\omega_{a}=0 \mid 1 \leq a \leq l\right\}$ on an $n$-dimensional manifold $M$, where $\omega_{a}$ for each $a$ is a differential 1-form. Then, the submanifold $N$ of $M$ is called an integral manifold of $\mathcal{P}$ if $\boldsymbol{i}_{\xi} \omega_{a}=0$ for any vector $\xi$ on the tangent space $T_{p} N$ at each point $p \in N$, where $1 \leq a \leq l$.

Definition 4. Let $M$ be an $n$-dimensional manifold $M$. A morphism associating an $r$-dimensional subspace $\Delta$ of the tangent space $T_{p} M$ with each point $p \in M$ is called an $r$-th order distribution. The distribution is called regular if the dimension $r$ is invariant. 
The integrability of $\mathcal{P}$ can be rephrased by distributions. That is, the $r$-dimensional distribution $\Delta$ is defined by $\Delta_{p}=\left\{\xi \in T_{p} M \mid \boldsymbol{i}_{\xi} \omega_{a}=0,1 \leq a \leq l\right\}$ for each point $p \in M$, where $r=n-l$.

\subsection{Symplectic Structure Restricted to Distributions}

The relationship between Lagrangian and Hamiltonian systems is given by Legendre transformations (see (65) in Section D). For classical field equations, the Legendre transformations (or Lagrangians) are not regular in general, and thus, they are not one-to-one. However, the Legendre transformation can reasonably work under the following weaker condition.

Proposition 1. [5] Let $P^{r-1}$ be an embedded closed subbundle of $Z^{r-1}$. Then, the Legendre transformation $\sigma_{\mathcal{L}}: J^{2 r-1} Q \rightarrow Z^{r-1}$ is called almost regular if $\sigma_{\mathcal{L}}$ is a submersion (i.e., $T \sigma_{\mathcal{L}}$ is surjective) with respect to the image $P^{r-1}$. In this case, there exists a vector field $\xi$ on $J^{2 r-1} M$ such that $T \sigma_{\mathcal{L}} \cdot \xi=v$ for any vector field $v$ on $P^{r-1}$.

Proposition 2. [5] The following conditions are equivalent:

1) $\phi \in \Gamma(Q)$ satisfies the Euler-Lagrange equations,

2) $j^{2 r-1} \phi \in \Gamma\left(J^{2 r-1} Q\right)$ satisfies the Cartan equations,

3) If $\sigma_{\mathcal{L}}$ is almost regular, $\delta=\sigma_{\mathcal{L}} \circ j^{2 r-1} \phi \in \Gamma\left(P^{r-1}\right)$ satisfies the Hamilton-De Donder equations,

where $\Gamma$ is the space of all sections, and $j^{2 r-1} \phi$ is the $(2 r-1)$-th jet of the section $\phi$.

Remark 1. Note that $\phi=\pi_{0}^{2 r-1} \circ \rho$ is not always the extremum of the original variational problem, i.e., Euler-Lagrange equations even if a certain $\rho \in \Gamma\left(J^{2 r-1} Q\right)$ is a Cartan equation, where $\pi_{0}^{2 r-1}: J^{2 r-1} Q \rightarrow Q$ is the natural projection. This correspondence is valid only if $\sigma_{\mathcal{L}}$ is regular [5]. Hence, we start from Euler-Lagrange equations in this paper.

Under the assumption of first order Lagrangians with respect to time, the covariant Legendre transformation $\sigma_{\mathcal{L}}: J^{2 r-1} Q \rightarrow Z^{r-1}$ in (65) is restricted the following instantaneous Legendre transformation:

$$
\begin{cases}p_{a}^{K t}=\frac{\partial \mathcal{L}}{\partial q_{K t}^{a}} & \text { if }|K t|=r, \\ p_{a}^{K t}=\frac{\partial \mathcal{L}}{\partial q_{K t}^{a}}-D_{s} p_{a}^{K t s} & \text { if } 1 \leq|K t|<r,\end{cases}
$$

where $0 \leq|K| \leq r-1$ is the multi index with respect to spatial variables, and $D_{s}=\sum_{i=1}^{m} D_{i}$ is the spatial total divergence. The term $-D_{s} p_{a}^{K t s}$ in the second equation of (12) is introduced from $p^{k t t}=0$ in $-D_{i} p_{a}^{K t i}$ of (65), because Lagrangian is first order with respect to time.

From the above preliminaries, we shall relate a distribution $\Delta_{Q}$ of Euler-Lagrange equations with a distribution $\Delta_{Z^{r-1}}$ of Hamiltonian systems on the multisymplectic manifold $Z^{r-1}$ through the relations i) $\rightarrow$ ii) $\rightarrow$ iii) in Proposition 2. Indeed, the following Hamiltonian representation for $\Delta_{Z^{r-1}}$ can be defined.

Definition 5. Consider the $(r-1)$-th multisymplectic manifold $Z^{r-1} Z^{r-1}$ with the local coordinates $\left(x^{i} ; q_{K}^{a}, p_{a}^{K t}, p\right)$ under Assumption 1. Then, the local coordinates of $V Z^{r-1}$ can be written as $\left(x^{i} ; q_{K}^{a}, p_{a}^{K t}, \delta q_{K}^{a}, \delta p_{a}^{K t}, p\right)$, because this results from the addition of the local coordinates of $Z^{r-1}$ and the coordinates $\left(\delta q_{K}^{a}, \delta p_{a}^{K t}\right)$ generated by the differential operator $\delta$ with respect to $q_{K}^{a}, p_{a}^{K t}$, and we have used the relations: $\delta q_{K}^{a}=D_{t} q_{K}^{a}=q_{K t}^{a}, \delta p_{a}^{K t}=D_{t} p_{a}^{K t}$. On the other hand, the local coordinates of $V^{*} Z^{r-1}$ are $\left(x^{i} ; q_{K}^{a}, p_{a}^{K t},-\delta p_{a}^{K t}, \delta q_{K}^{a}, p\right)$, because it is given by the pairing of $-\mathrm{d} \Theta \in V^{*} Z^{r-1}$ and arbitrary vector $\xi \in V Z^{r-1}$ that is defined by $\boldsymbol{i}_{\xi}(-\mathrm{d} \Theta)$ between the vertical tangent and cotangent bundles [1]. Note that there is no adjoint variable of $p$ defining an affine structure [5] (p. 214).

Now, we can consider the following induced symplectic structure induced from distributions.

Proposition 3. Let $\sigma_{\mathcal{L}}: J^{2 r-1} Q \rightarrow Z^{r-1}$ be the almost regular Legendre transformation and let $\Delta_{Q} \subset V Q$ be a regular distribution on $Q$ that is restricted to $\mathcal{S}$. We restrict the multisymplectic $(m+2)$-form $\Omega$ over $Z^{r-1}$ (see Section D) to $\Delta_{Z^{r-1}}$, i.e., we define a skew symmetric bilinear form by $\Omega_{\Delta}=\left.\Omega\right|_{\Delta_{Z^{r-1}} \times \Delta_{Z^{r-1}}}$. Then, there 
exists the following subbundle $\mathbb{D}_{\Delta} \subset V Z^{r-1} \oplus V^{*} Z^{r-1}$ for each $z \in Z^{r-1}$ and a fixed $p$ :

$$
\mathbb{D}_{\Delta}(z)=\left\{\left(v_{z}, \alpha_{z}\right) \in V_{z} Z^{r-1} \times V_{z}^{*} Z^{r-1} \mid v_{z} \in \Delta_{Z^{r-1}}(z), \alpha_{z}\left(w_{z}\right)=\Omega_{\Delta}(z)\left(v_{z}, w_{z}\right), \forall w_{z} \in \Delta_{z^{r-1}}(z)\right\},
$$

where we have defined $\Delta_{Z^{r-1}}=V \sigma_{\mathcal{L}} \circ\left(V \pi_{0}^{2 r-1}\right)^{-1} \Delta_{Q} \subset V Z^{r-1}, V \sigma_{\mathcal{L}}$ is the vertical tangent map of $\sigma_{\mathcal{L}}$, and $\left(V \pi_{0}^{2 r-1}\right)^{-1} \Delta_{Q} \subset V J^{2 r-1} Q$ is the distribution lifted along the vertical tangent map $V \pi_{0}^{2 r-1}: V J^{2 r-1} Q \rightarrow V Q$ of the canonical projection $\pi_{0}^{2 r-1}: J^{2 r-1} Q \rightarrow Q$.

Proof. According to Proposition 2, there exists a vector field $\xi \in T J^{2 r-1} Q$ for any vector field $v \in \Delta_{Z^{r-1}}$. Thus, there exists also $\zeta=\varpi_{2 r-1} \xi$, where $\varpi_{2 r-1}: T J^{2 r-1} Q \rightarrow V J^{2 r-1} Q$ is the natural projection. Hence, the relation in (13) is resulted from the nature of the symplectic structure. Indeed, for a given $v_{z} \in \Delta_{Z^{r-1}}(z) \subset V_{z} Z^{r-1}$, there always exists the corresponding $(m+1,1)$-form $\alpha_{z} \in V_{z}^{*} Z^{r-1}$ that determines a Hamiltonian vector field.

Let us introduce Dirac structures on vector spaces form the references [2] [18] [19].

Definition 6. A Dirac structure on a vector space $A$ is a subspace $\mathbb{D} \subset A \oplus A^{*}$ such that $\mathbb{D}=\mathbb{D}^{\perp}$, where $A^{*}$ is the dual space of $\mathrm{A}, \mathbb{D}^{\perp}$ is the orthogonal space of $\mathbb{D}$ with respect to the symmetric pairing $\langle\langle\cdot, \cdot\rangle\rangle$ on $A \oplus A^{*}$ such that $\langle\langle(v, \alpha),(\bar{v}, \bar{\alpha})\rangle\rangle=\langle\alpha, \bar{v}\rangle+\langle\bar{\alpha}, v\rangle$ for $((v, \alpha),(\bar{v}, \bar{\alpha})) \in A \oplus A^{*}$, and $\langle\cdot, \cdot\rangle$ is the natural pairing between $A^{*}$ and $A$.

Corollary 1. $\mathbb{D}_{\Delta}$ in (13) is the (almost) Dirac structure.

Proof. If we fix the coordinate $p$ of $Z^{r-1}$, i.e., the covariant Hamiltonian, then Equation (13) is the typical form of induced Dirac structures [15].

\section{Distirubted Port-Hamiltonian Systems with Higher Order Boundary Energy Flows}

In this section, we derive a formal structure of distributed port-Hamiltonian systems with boundary energy flows including higher order derivatives from the previously discussed implicit Hamiltonian representation. The energy flows passing through boundaries of system domains are used for boundary interconnections, or passivitybased boundary controls.

\subsection{Boundary Terms Generated by Integration by Parts}

In higher order variational problems, the zero boundary condition is usually assumed for simplification or some other reason. Then, boundary terms generated by Stokes theorem after applying integration by parts are eliminated. Actually, these boundary terms are related with the boundary energy flows.

Let us recall such a calculation that yields the boundary term in variational calculus. We first define the following notation for simplification.

Definition 7. From the Legendre transformation $\sigma_{\mathcal{L}}$ in (65), we can derive the following variable:

$$
\tilde{p}_{a}^{I}:=\frac{\partial \mathcal{L}}{\partial q_{I}^{a}}=p_{a}^{I}+D_{i} p_{a}^{I i},
$$

where $0 \leq|I| \leq r, \quad 0 \leq i \leq m, D_{i}$ is the total differential operator (61), and we have set $p_{a}^{I i}=0$ for $|I|=r$.

Now, we consider the Lagrangian density functional $\int_{\mathcal{Y}} \mathcal{L} \mathrm{d} x$. The variational derivative of the Lagrangian density functional can be transformed by the integration by parts formula as follows:

$$
\int_{\mathcal{Y}} \frac{\partial \mathcal{L}}{\partial q_{K i}^{a}} \mathrm{~d} q_{K i}^{a} \wedge \mathrm{d} x=\int_{\mathcal{Y}} \tilde{p}_{a}^{K i} \mathrm{~d} q_{K i}^{a} \wedge \mathrm{d} x=\int_{\mathcal{Y}} D_{i}\left(\tilde{p}_{a}^{K i} \mathrm{~d} q_{K}^{a}\right) \wedge \mathrm{d} x-\int_{\mathcal{Y}} D_{i} \tilde{p}_{a}^{K i} \mathrm{~d} q_{K}^{a} \wedge \mathrm{d} x,
$$

where $0 \leq|K| \leq r-1$. By Stokes theorem [22], for any $0 \leq i \leq m$, the first term in the right side of (15) can be transformed into

$$
\int_{\mathcal{Y}} D_{i}\left(\tilde{p}_{a}^{K i} \mathrm{~d} q_{K}^{a}\right) \wedge \mathrm{d} x=\int_{\mathcal{Y}} \tilde{p}_{a}^{K i} \mathrm{~d} q_{K}^{a} \wedge \boldsymbol{i}_{\partial x^{i}} \mathrm{~d} x
$$

where $D_{i}$ is the total divergence that acts as $\sum_{i=0}^{m} D_{i}$, and $\boldsymbol{i}_{\partial x^{i}} \mathrm{~d} x$ is the volume $(m, 1)$-form on $\partial \mathcal{Y}$. For the 
integrands in (15) and (16), consider the operation of differential forms that separate a coefficient from a vertical basis. For example, $\tilde{p}_{a}^{K i} \mathrm{~d} q_{K i}^{a} \wedge \mathrm{d} x$ can be decomposed into $\tilde{p}_{a}^{K i}$ and $\mathrm{d} q_{K i}^{a}$. This operation is defined as follows.

Definition 8. For an $(m+1,1)$-form $\mathrm{d}_{v} \mathcal{L} \mathrm{d} x=\tilde{p}_{a}^{K i} \mathrm{~d} q_{K i}^{a} \mathrm{~d} x \in \Omega^{m+1,1}\left(J^{2 r-1} Q\right)$, we define the integration by parts operator as the following local expression:

$$
\begin{aligned}
& \mathbb{I}(|K i|)(\mathrm{d} \mathcal{L}):\left.\left.\Omega^{m+1,1}\left(J^{2 r-1} Q\right)\right|_{\mathcal{Y}} \rightarrow \Omega^{m+1,1}\left(J^{2 r-1} Q\right)\right|_{\mathcal{Y} \times \partial \mathcal{Y}} ;\left(x^{i} ;{ }^{k}, q_{K i}^{a},{ }^{2 r-1}, \tilde{p}_{a}^{K i}, \cdots\right) \\
& \mapsto\left\{\left(x^{i} ; \cdots, q_{K}^{k-1}, \cdots,-D_{i} \tilde{p}_{a}^{K i}, \cdots\right),\left(x^{i} ; \cdots, \cdots, q_{K}^{a}, \cdots, \tilde{p}_{a}^{K i}, \cdots\right)\right\},
\end{aligned}
$$

where we denoted $\left.\Omega^{m+1,1}\left(J^{2 r-1} Q\right)\right|_{\mathcal{Y} \times \partial \mathcal{Y}}=\left.\Omega^{m+1,1}\left(J^{2 r-1} Q\right)\right|_{\mathcal{Y}} \times\left.\Omega^{m, 1}\left(J^{2 r-1} Q\right)\right|_{\partial \mathcal{Y}}$, i.e., the style $\{(A),(B)\}$ means the set of the transformed local coordinates $(A)$ on $\mathcal{Y}$ and $(B)$ on $\partial \mathcal{Y}$, respectively. Here, untransformed coordinates under $|K i|$-th order are omitted in (17), and their numbers can be explicitly calculated by $k=|K|$, $0 \leq k \leq r-1$ and $j=r-k$.

Remark 2. For a Lagrangian density $\mathcal{L} \mathrm{d} x^{s} \in \Omega^{m, 1}\left(J^{2 r-1} Q\right)$ restricted to the spatial domain $\mathcal{S}$ in the timespatial split space, the operation (17) can be also well defined. In this case, the boundary terms generated by Stokes theorem with respect to the time derivative $D_{t}(\cdot)$ in the total divergence are eliminated, because there is no boundary of a point in the time axis.

The repeated application of the integration by parts operator can yield all variations of boundary terms appeared in variational calculus.

Proposition 4. For some I, where $1 \leq v \leq|I|$, the $v$-th degree integration by parts operators is defined by

$$
\mathbb{I}^{v}(|I|)(\mathrm{d} \mathcal{L})=\mathbb{I}(|I|-v+1) \circ \cdots \circ \mathbb{I}(|I|-1) \circ \mathbb{I}(|I|)(\mathrm{d} \mathcal{L})
$$

that can be expressed as the coordinate transformation

$$
\begin{cases}p_{\partial a}^{K i}=0 & \text { if }|K i|=r \\ p_{\partial a}^{K}=\frac{\partial \mathcal{L}}{\partial q_{K i}^{a}}-D_{i} p_{\partial a}^{K i} & \text { if } 0 \leq|K|<r .\end{cases}
$$

Proof. From the direct calculation of $\mathbb{I}^{v}(|I|)(\mathrm{d} \mathcal{L})$, we obtain the following representation on $\left.\Omega^{m+1,1}\left(J^{2 r-1} Q\right)\right|_{\mathcal{Y} \times \partial \mathcal{Y}}$ that is derived from that on $\left.\Omega^{m+1,1}\left(J^{2 r-1} Q\right)\right|_{\mathcal{Y}}$ :

$$
\left\{\left(x^{i} ; q_{J}^{a}, \sum_{|I|=0}^{r}\left(-D_{i}\right)^{|I|} \tilde{p}_{a}^{I}, 0, \ldots, 0\right),\left(x^{i} ; q_{J}^{a}, \sum_{|I|=1}^{r}\left(-D_{i}\right)^{|I|-1} \tilde{p}_{a}^{I}, \cdots, \sum_{|I|=i}^{r}\left(-D_{i}\right)^{|I|-i} \tilde{p}_{a}^{I}, \cdots, \tilde{p}_{a}^{I-1}-D_{i} \tilde{p}_{a}^{I}, \tilde{p}_{a}^{I}, 0\right)\right\} .
$$

The first bracket of (20) includes the nonzero part of the Euler-Lagrange equation; therefore, this operation is equivalent to the variational differential. The last $r$ elements in the second bracket correspond to $p_{\partial a}^{K}$ in (19) for $0 \leq|K| \leq r$.

\subsection{Stokes Variational Differential}

The symplectic structure induced from distributions does not have any information on boundary energy flows. In this section, we define a variational differential operator with boundary terms generated by integration by parts and Stokes theorem, called the Stokes variational differential. The Stokes variational differential can be used in the induced symplectic structure for relating a given Lagrangian with port-Hamiltonian representations.

Proposition 5. For an $r$-th order Lagrangian density $\mathcal{L} \in \Omega^{m+1,0}\left(J^{r} Q\right)$ that is first order with respect to time and $(r-1)$-th order with respect to spatial coordinates, the following variational differential operation can be defined:

$$
\mathfrak{d}^{r} \mathcal{L}=\chi_{J^{r-1} Q} \circ \mathbb{I}^{r}(r)\left(\left.d\left(l_{1, r-1}^{r} \mathcal{L}\right)\right|_{\mathcal{S}}\right):\left.\left.\Omega^{m+1,0}\left(J^{r} Q\right)\right|_{\mathcal{S}} \rightarrow V^{*} Z^{r-1}\right|_{\mathcal{S} \times \mathcal{O} \mathcal{S}}
$$


where $\chi_{J^{r-1} Q}: V^{*} J^{1} J^{r-1} Q \rightarrow V^{*} Z^{r-1}$ (given as $\Omega_{Z}^{b} \circ \kappa_{\star}^{-1}$ ). We call $\mathfrak{d}^{r}$ the Stokes variational differential on $\mathcal{S}$ with $\partial \mathcal{S}$.

Before the proof of Proposition 5, we should prepare the following bundle maps. We first consider the map between $V Z^{r-1}$ and $V^{*} Z^{r-1}$ over $Z^{r-1}$.

Lemma 1. We can define the following bundle map $\Omega_{Z}^{b}$ :

$$
\begin{aligned}
& \Omega_{Z}^{b}: V Z^{r-1} \rightarrow V^{*} Z^{r-1} ; \\
& \left(x^{i} ; q_{K}^{a}, p_{a}^{K i}, \delta q_{K}^{a}, \delta p_{a}^{K i}, p\right) \mapsto\left(x^{i} ; q_{K}^{a}, p_{a}^{K i},-\delta p_{a}^{K i}, \delta q_{K}^{a}, p\right) .
\end{aligned}
$$

Proof. This can be proven by the direct calculation with respect to the symplectic form (see Definition 5).

Lemma 2. By using the Legendre transformation $\sigma_{\mathcal{L}}: J^{2 r-1} Q \rightarrow Z^{r-1}$, we can define the following bundle map under the first order assumption:

$$
\begin{aligned}
& \kappa_{\star}^{-1}: V^{*} J^{1} J^{r-1} Q \rightarrow V Z^{r-1} ; \\
& \left(x^{i} ; q_{K}^{a}, \delta q_{K}^{a}, p_{a}^{K t}, \delta p_{a}^{K t}\right) \mapsto\left(x^{i} ; q_{K}^{a}, \delta p_{a}^{K t}, \delta q_{K}^{a}, p_{a}^{K t}, p\right),
\end{aligned}
$$

where $1 \leq|K t| \leq r$, and $p$ is defined by $\sigma_{\mathcal{L}}$.

Proof. There exists the following bundle map $\kappa_{J^{r-1} Q}$ under the assumption:

$$
\begin{aligned}
& \kappa_{J^{r-1} Q}: V V^{*} J^{r-1} Q \rightarrow V^{*} V J^{r-1} Q ; \\
& \left(x^{i} ; q_{K}^{a}, p_{a}^{K t}, \delta q_{K}^{a}, \delta p_{a}^{K t}\right) \mapsto\left(x^{i} ; q_{K}^{a}, \delta q_{K}^{a}, \delta p_{a}^{K t}, p_{a}^{K t}\right) .
\end{aligned}
$$

This can be proven in analogy with the diffeomorphism in the lumped parameter case, i.e., $\kappa_{Q}: T T^{*} Q \rightarrow$ $T^{*} T Q ; \quad(x ; q, p, \delta q, \delta p) \mapsto(x ; q, \delta q, \delta p, p) \quad$ ([16], p. 140, [26]). The inverse map of (24) can be extended as a map on the affine bundle (23). Indeed, we have the bundle map between the bundle $V^{*} J^{1} J^{r-1} Q$ and the original bundle $V J^{1^{*}} J^{r-1} Q$ of the tangent affine bundle $V Z^{r-1} \cong V J^{1 \star} J^{r-1} Q$. Because the local coordinates of $J^{1^{*}} J^{r-1} Q$ are $\left(x^{i} ; q_{K}^{a}, p_{a}^{K i}\right)$, in the case of first order with respect to time, the local coordinates of $J^{1^{*}} J^{r-1} Q$ are $\left(x^{i} ; q_{K}^{a}, p_{a}^{K t}\right)$, and those of $J^{1} J^{r-1} Q$ are $\left(x^{i} ; q_{K}^{a}, q_{K t}^{a}\right)$. Thus, under the assumption, we can regard $V J^{1^{*}} J^{r-1} Q$ and $V^{*} J^{1} J^{r-1} Q$ as, respectively, $V V^{*} J^{r-1} Q$ and $V^{*} V J^{r-1} Q$ by identifying $J^{1} J^{r-1} Q$ and $V J^{r-1} Q$.

Next, we regard the derivative of Lagrangian density $\mathrm{d} \mathcal{L}$ as a differential $(m+1,1)$-form on $J^{1} J^{r-1} Q$ in the above discussion by using $J^{r} Q \hookrightarrow J^{1} J^{r-1} Q$.

Lemma 3. The standard variational derivative

$$
\begin{aligned}
& \mathrm{d}_{v} \circ \imath_{r}^{2 r-1}: \Omega^{m+1,0}\left(J^{r} Q\right) \rightarrow \Omega^{m+1,1}\left(J^{2 r-1} Q\right) ; \\
& \mathcal{L} \mathrm{d} x \mapsto \frac{\partial \mathcal{L}}{\partial q_{I}^{a}} \mathrm{~d} q_{I}^{a} \wedge \mathrm{d} x
\end{aligned}
$$

can be regarded as the following derivative under the assumption of first order Lagrangians with respect to time:

$$
\begin{aligned}
& \mathrm{d}_{v} \circ \gamma_{1, r-1}^{r}: \Omega^{m+1,0}\left(J^{1} J^{r-1} Q\right) \rightarrow V^{*} J^{1} J^{r-1} Q ; \\
& \mathcal{L} \mathrm{d} x \mapsto\left(\tilde{p}_{a}^{K} \mathrm{~d} q_{K}^{a}+\tilde{p}_{a}^{K t} \mathrm{~d} q_{K t}^{a}\right) \wedge \mathrm{d} x,
\end{aligned}
$$

where we have defined the inclusions $r_{2 r-1}^{r}: J^{r} Q \hookrightarrow J^{2 r-1} Q ;\left(x^{i} ; q_{K}^{a}\right) \mapsto\left(x^{i} ; q_{K K i}^{a}\right)$, and $u_{1, r-1}^{r}: J^{r} Q \hookrightarrow J^{1} J^{r-1} Q ;\left(x^{i} ; q_{K}^{a}\right) \mapsto\left(x^{i} ; q_{K}^{a}, q_{K i}^{a}\right)$

Proof. By $l_{1,2 r-2}^{2 r-1}: J^{2 r-1} Q \hookrightarrow J^{1} J^{2 r-2} Q ; \quad\left(x^{i} ; q_{K K i}^{a}\right) \mapsto\left(x^{i} ; q_{K K}^{a}, q_{K K i}^{a}\right)$, and $l_{1,2 r-2}^{1, r-1}: J^{1} J^{r-1} Q \hookrightarrow J^{1} J^{2 r-2} Q ;$ $\left(x^{i} ; q_{K}^{a}, q_{K i}^{a}\right) \mapsto\left(x^{i} ; q_{K K}^{a}, q_{K K i}^{a}\right)$, we can rewrite (25) as

$$
\mathrm{d}_{v} \circ l_{1,2 r-2}^{1, r-1}: \Omega^{m+1,0}\left(J^{1} J^{r-1} Q\right) \rightarrow V^{*} J^{1} J^{2 r-2} Q ; \quad \mathcal{L} \mathrm{d} x \mapsto\left(\tilde{p}_{a}^{K} \mathrm{~d} q_{K}^{a}+\tilde{p}_{a}^{K i} \mathrm{~d} q_{K i}^{a}\right) \wedge \mathrm{d} x,
$$


where we have used $\Omega^{m+1,1}(Y) \cong V^{*} Y$. Moreover, the inclusion $l_{1,2 r-2}^{1, r-1}$ yields the dual map

$$
\begin{aligned}
& V^{*}{ }_{1,2 r-2}^{1, r-1}: V^{*} J^{1} J^{2 r-2} Q \rightarrow V^{*} J^{1} J^{r-1} Q ; \\
& \left(x^{i} ; q_{K K}^{a}, \delta q_{K K}^{a}, p_{a}^{K K i}, \delta p_{a}^{K K i}\right) \mapsto\left(x^{i} ; q_{K}^{a}, \delta q_{K}^{a}, p_{a}^{K i}, \delta p_{a}^{K i}\right) .
\end{aligned}
$$

Under the assumption, we can specify by $i=t$ in the above equations.

Proof of Proposition 5: From Lemmas 1-3, we can see that the map $\mathfrak{d}^{r}$ is well-defined.

\subsection{Local Expression of Induced Symplectic Structures}

From the previous preparations, we can derive the relationship between the distribution $\Delta_{Q}$ and an instantaneous Hamiltonian system on $Z^{r-1}$ that is described by the induced symplectic structure $\mathbb{D}_{\Delta}$ using the Stokes variational differential $\mathfrak{d}^{r} \mathcal{L}$.

Definition 9. Let $\mathcal{L}=\mathcal{L} \mathrm{d} x \in \Omega^{m+1,0}\left(J^{r} Q\right)$ be a Lagrangian density that is first order with respect to time, and let $\Delta_{Q} \subset V Q$ be a regular distribution on $Q$. Consider the symplectic structure $\mathbb{D}_{\Delta}$ induced on $Z^{r-1}$ in (13), and the Stokes variational differential $\mathfrak{d}^{r} \mathcal{L}:\left.\left.\Omega^{m+1,0}\left(J^{r} Q\right)\right|_{\mathcal{S}} \rightarrow V^{*} Z^{r-1}\right|_{\mathcal{S} \times \partial \mathcal{S}}$ on $\mathcal{S}$ with $\partial \mathcal{S}$ in (21). Let $P_{\Delta}=V \sigma_{\mathcal{L}}\left(\Delta_{J^{2 r-1} Q}\right) \subset V Z^{r-1}$ be the image of $\Delta_{Q}$, where we have defined $\Delta_{J^{2 r-1} Q}=\left(V \pi_{0}^{2 r-1}\right)^{-1} \Delta_{Q}$. Then, we define the implicit higher order field Hamiltonian representation on $\mathcal{S}$ with $\partial \mathcal{S}$ as

$$
\left(\mathfrak{X}_{Z^{r-1}}, \mathfrak{d}^{r} \mathcal{L}\right) \in \mathbb{D}_{\Delta},
$$

where $\mathfrak{X}_{Z^{r-1}} \in P_{\Delta}$.

Theorem 1. The local expression of the implicit higher order field port Hamiltonian representation on $\mathcal{S}$ with $\partial \mathcal{S}$ determined by $\left(\mathfrak{X}_{Z^{r-1}}, \mathfrak{d}^{r} \mathcal{L}\right) \in \mathbb{D}_{\Delta}$ is given as follows:

$$
\left\{\begin{array}{l}
D_{t} q_{K}^{a} \in \Delta_{Z^{r-1}}, \quad q_{K t}^{a}=D_{t} q_{K}^{a}, \quad(-1)^{|K|} D_{K}\left(D_{t} \tilde{p}_{a}^{K t}-\tilde{p}_{a}^{K}\right) \in \Delta_{Z^{r-1}}^{\circ}, \\
\tilde{p}_{a}^{K t}=\frac{\partial \mathcal{L}}{\partial q_{K t}^{a}}, \quad \tilde{p}_{a}^{K}=\frac{\partial \mathcal{L}}{\partial q_{K}^{a}},
\end{array}\right.
$$

where $\tilde{p}_{a}^{K}$ is the covariant momentum, and we have defined the null space $\Delta_{\mathrm{Z}^{r-1}}^{\circ}=\left\{\alpha \in V^{*} Z^{r-1} \mid\langle\alpha, v\rangle=0, \forall v \in P_{\Delta} \subset V Z^{r-1}\right\}$. On the boundary, there is the following $(m, 1)$-form:

$$
\left.\left(-D_{s}\right)^{j-1} \frac{1}{w(K)} \tilde{p}_{a}^{\bar{K}} \cdot D_{s}^{|\bar{K}|-j} \mathrm{~d} q_{t}^{a}\right|_{\partial \mathcal{S}},
$$

where $1 \leq j \leq|K|, K$ is the multi index with respect to spatial coordinates, $\bar{K}$ is the multi index generated by repeated permutations (see Section $B$ ), and we have defined the spatial total divergence $D_{s}=\sum_{i=1}^{m} D_{i}$.

Proof. Let $\mathfrak{X}_{Z^{r-1}}=\left(x^{i} ; q_{K}^{a}, p_{a}^{K t}, D_{t} q_{K}^{a}, D_{t} p_{a}^{K t}, p\right)$ be the vector field on $V Z^{r-1}$. For a vector field $\mathfrak{X}_{Z^{r-1}}$, we consider the following local expression of the symplectic form $\Omega$

$$
\Omega\left(\left(x^{i}, q_{K}^{a}, p_{a}^{K t}, u^{\prime}, \alpha^{\prime}, p\right),\left(x^{i}, q_{K}^{a}, p_{a}^{K t}, u, \alpha, p\right)\right)=\left\langle\alpha, u^{\prime}\right\rangle-\left\langle\alpha^{\prime}, u\right\rangle,
$$

where $\langle\alpha, u\rangle: V Z^{r-1} \times V^{*} Z^{r-1} \rightarrow \mathbb{R}$ is the natural pairing between $u \in \mathfrak{X}_{Z^{r-1}} \subset V Z^{r-1}$ and $\alpha \in V^{*} Z^{r-1}$. On the other hand, from the local expression of the Stokes variational differential on $\mathcal{S}$

$$
\mathfrak{d}^{r} \mathcal{L}=\left(x^{i} ; q_{K}^{a}, \tilde{p}_{a}^{K t},-\delta \tilde{p}_{a}^{K t}, \delta q_{K}^{a}, p\right),
$$

we denote the 1 -form fields by $u^{*}:=-\delta \tilde{p}_{a}^{K t}$ and $\alpha^{*}:=\delta q_{K}^{a}$. From the equivalent condition between the last relation in (13) and (32)

$$
\left\langle u^{*}, u\right\rangle+\left\langle\alpha^{*}, \alpha\right\rangle=\left\langle\alpha, u^{\prime}\right\rangle-\left\langle\alpha^{\prime}, u\right\rangle
$$

we obtain the condition 


$$
\left\langle-\delta \tilde{p}_{a}^{K t}, u\right\rangle+\left\langle q_{K t}^{a}, \alpha\right\rangle=\left\langle\alpha, D_{t} q_{K}^{a}\right\rangle-\left\langle D_{t} \tilde{p}_{a}^{K t}, u\right\rangle
$$

for any $u$ and $\alpha$. Then, Equation (35) yields (30), because $\tilde{p}_{a}^{K t}=\partial \mathcal{L} / \partial q_{K t}^{a}$, and $\delta \tilde{p}_{a}^{K t}=\partial \mathcal{L} / \partial q_{K}^{a}$ that can be derived from the Euler-Lagrange equation. Indeed, the pairing with respect to $u$ gives the third relation in (30) as follows:

$$
\left(D_{t} \tilde{p}_{a}^{K t}-\frac{\partial \mathcal{L}}{\partial q_{K}^{a}}\right) \delta q_{K}^{a}=(-1)^{|K|} D_{K}\left(D_{t} \tilde{p}_{a}^{K t}-\frac{\partial \mathcal{L}}{\partial q_{K}^{a}}\right) \delta q^{a}+\Phi,
$$

where $\Phi$ corresponds to the boundary term in (31). The term (31) is obtained from the calculation in (20). Here, the total divergence $D_{t}(\cdot)$ with respect to time has been eliminated. Consequently, in (30), the first relation means a given vector field, the second relation is the definition of jet variables, the third relation is the Euler-Lagrange equation before applying integration by parts, and the fourth relation is the definition of the momentum.

The above representation can be converted to the following formal form of port representations [7].

Corollary 2. The implicit higher order field port Hamiltonian system defined on $\mathcal{S}$ with $\partial \mathcal{S}$ can be rewritten as the following port Hamiltonian system:

$$
\left[\begin{array}{c}
f_{p}^{a} \\
f_{q K}^{a}
\end{array}\right]=\left[\begin{array}{cc}
0 & -(-1)^{|K|} D_{K} \\
D_{K}^{\mathrm{T}} & 0
\end{array}\right]\left[\begin{array}{c}
e_{p}^{a} \\
e_{q K}^{a}
\end{array}\right],
$$

where $1 \leq a \leq l$, and we have defined the variables

$$
\left\{\begin{array}{l}
\left(f_{p}^{a}, e_{p}^{a}\right)=\left((-1)^{|K t|} D_{K t} \tilde{p}_{a}^{K t}, q_{t}^{a}\right), \quad\left(f_{q K}^{a}, e_{q K}^{a}\right)=\left(D_{t} q_{K}^{a}, \tilde{p}_{a}^{K}\right), \\
\left(f_{\partial|\bar{K}|, j}^{a}, e_{\partial|\bar{K}|, j}^{a}\right)=\left(\left.D_{s}^{|\bar{K}|-j} e_{p}^{a}\right|_{\partial \mathcal{S}},-\left.\left(-D_{s}\right)^{j-1} e_{q \bar{K}}^{a}\right|_{\partial \mathcal{S}}\right) \\
\tilde{p}_{a}^{K t}=\frac{\partial \mathcal{L}}{\partial q_{K t}^{a}}, \quad \tilde{p}_{a}^{K}=\frac{\partial \mathcal{L}}{\partial q_{K}^{a}}
\end{array}\right.
$$

for $1 \leq j \leq|K|$. We call $f_{\partial \mid \bar{K}, j}^{a}$ and $e_{\partial|\bar{K}|, j}^{a}$ boundary port variables.

Proof. Form the third and second relations in (30), we can obtain the first and second rows in (37), respectively. The product of the pair of the third relation in (38) that is equivalent to the boundary term in (31), where $\mathrm{d} q_{t}^{a}$ in (31) has been interpreted as an infinitesimal variation of $q_{t}^{a}$.

\subsection{Power Balance and Passivity}

This section derives the power balance of the Hamiltonian representation discussed in the previous section, and define the formal representation of higher order field port Hamiltonian systems with boundary energy flows.

In the time-spatial split space, the instantaneous Hamiltonian (70) on $\mathcal{S}$ is given by

$$
\mathscr{H}_{\mathcal{S}}=\int_{\mathcal{S}}\left(\sum_{|K|=0}^{r-1} p_{a}^{K t} q_{K t}^{a}-\mathcal{L}\right) \mathrm{d} x^{s},
$$

where $1 \leq j \leq|K|$. The following relation corresponds to the power balance of distributed port-Hamiltonian systems.

Proposition 6. The system (37) satisfies the power balance

$$
\int_{\mathcal{S}}\left(e_{p}^{a} f_{p}^{a}+e_{q K}^{a} f_{q K}^{a}\right) \mathrm{d} x^{s}+\int_{\partial \mathcal{S}}\left(\sum_{j=1}^{|\bar{K}|} e_{\partial|\bar{K}|, j}^{a} f_{\partial|\bar{K}|, j}^{a}\right) \boldsymbol{i}_{\partial x^{S}} \mathrm{~d} x^{s}=0 .
$$

Proof. The power balance can be derived from the interior product between the derivative of the instantaneous Hamiltonian (39) and arbitrary vector field is zero. Let $\mathcal{H}\left(x, q_{I}^{a}, p_{a}^{K t}\right)=p_{a}^{K t} q_{K t}^{a}-\mathcal{L}\left(x, q_{I}^{a}\right)$ be the generalized energy density of (39) defined on $\Omega_{v}^{0}\left(Z^{r-1} \oplus J^{2 r-1} M\right)$, where $0 \leq|I| \leq r$, and $Z^{r-1} \oplus J^{2 r-1} Q$ is the Whitney 
bundle with the local coordinates $\left(x^{i} ; q_{J}^{a}, p_{a}^{K i}, p\right)$ for $0 \leq|K| \leq r-1$ and $0 \leq|J| \leq 2 r-1$. Next, consider the pairing between $\mathrm{d}_{v} \mathcal{H}$ and the vector field $\mu=D_{t} q_{J}^{a}\left(\partial / \partial q_{J}^{a}\right)+D_{t} p_{a}^{K t}\left(\partial / \partial p_{a}^{K t}\right) \in V\left(Z^{r-1} \oplus J^{2 r-1} M\right)$ as follows:

$$
\begin{aligned}
\frac{\mathrm{d} \mathscr{K} \mathcal{S}}{\mathrm{d} t} & =\boldsymbol{i}_{\mu} \mathrm{d}_{v} \mathcal{H}=\boldsymbol{i}_{\mu} \mathrm{d}_{v}\left(p_{a}^{K t} q_{K t}^{a}-\mathcal{L}\right)=\boldsymbol{i}_{\mu}\left(p_{a}^{K t} \mathrm{~d} q_{K t}^{a}+q_{K t}^{a} \mathrm{~d} p_{a}^{K t}-\frac{\partial \mathcal{L}}{\partial q_{I}^{a}} \mathrm{~d} q_{I}^{a}\right) \\
& =\boldsymbol{i}_{\mu}\left(-D_{s} p_{a}^{K t s} \mathrm{~d} q_{K t}^{a}+q_{K t}^{a} \mathrm{~d} p_{a}^{K t}-\frac{\partial \mathcal{L}}{\partial q_{K}^{a}} \mathrm{~d} q_{K}^{a}\right) \\
& =\boldsymbol{i}_{\mu}\left(D_{t} D_{s} p_{a}^{K t s} \mathrm{~d} q_{K}^{a}+q_{K t}^{a} \mathrm{~d} p_{a}^{K t}-\frac{\partial \mathcal{L}}{\partial q_{K}^{a}} \mathrm{~d} q_{K}^{a}\right) \\
& =\left(D_{t} D_{s} p_{a}^{K t s}+D_{t} p_{a}^{K t}-\frac{\partial \mathcal{L}}{\partial q_{K}^{a}}\right) q_{K t}^{a}=\left(D_{t} \tilde{p}_{a}^{K t}-\frac{\partial \mathcal{L}}{\partial q_{K}^{a}}\right) q_{K t}^{a},
\end{aligned}
$$

where we have used $\tilde{p}_{a}^{K t}=p_{a}^{K t}+D_{s} p_{a}^{K t s}=\partial \mathcal{L} / \partial q_{K t}^{a}$, and the time total divergence $D_{t}(\cdot)$ is eliminated in the same way of the proof of Theorem 1 . From the energy conservation $\boldsymbol{i}_{\mu} \mathrm{d}_{v} \mathcal{H}=0$, we obtain $D_{t} \tilde{p}_{a}^{K t}=\partial \mathcal{L} / \partial q_{K}^{a}$. On the other hand, we have

$$
\begin{aligned}
\boldsymbol{i}_{\mu} \mathrm{d}_{v} \mathcal{L} & =\boldsymbol{i}_{\mu}\left[\frac{\partial \mathcal{L}}{\partial q_{K t}^{a}} \mathrm{~d} q_{K t}^{a}+\frac{\partial \mathcal{L}}{\partial q_{K}^{a}} \mathrm{~d} q_{K}^{a}\right] \\
& =\boldsymbol{i}_{\mu}\left[-D_{t} \tilde{p}_{a}^{K t} \mathrm{~d} q_{K}^{a}+(-1)^{|K|} D_{K} \frac{\partial \mathcal{L}}{\partial q_{K}^{a}} \mathrm{~d} q^{a} \sum_{j=1}^{|\bar{K}|} D_{s}\left(\left(-D_{s}\right)^{j-1} \frac{1}{w(K)} \frac{\partial \mathcal{L}}{\partial q_{\bar{K}}^{a}} \cdot D_{s}^{|\bar{K}|-j} q_{t}^{a}\right)\right] \\
& =-D_{t} \tilde{p}_{a}^{K t} q_{K t}^{a}-(-1)^{|K t|} D_{K t} \tilde{p}_{a}^{K t} q_{t}^{a}-D_{s} \sum_{j=1}^{|\bar{K}|} e_{\partial|\bar{K}|, j}^{a} f_{\partial|\bar{K}|, j}^{a}=0 .
\end{aligned}
$$

By substituting (42) into (41), the integrand of (40) is given as follows:

$$
-\boldsymbol{i}_{\mu} \mathrm{d}_{v} \mathcal{H}=(-1)^{|K t|} D_{K t} \tilde{p}_{a}^{K t} q_{t}^{a}+\frac{\partial \mathcal{L}}{\partial q_{K}^{a}} q_{K t}^{a}+D_{s} \sum_{j=1}^{|\bar{K}|} e_{\partial|\bar{K}|, j}^{a} f_{\partial|\bar{K}|, j}^{a}=0 .
$$

By applying Stokes theorem to the integral of the third term of the second equation in (43), (40) is given.

Proposition 7. The system (37) is passive.

Proof. The Hamiltonian (39) and the power balance (40) correspond to the finite constant and the duality product before the time integration, respectively, in the definition of the passivity (see Section A).

Consequently, we at last reach the final result that means the system (37) is just a higher order representation of distributed port-Hamiltonian systems.

Theorem 2. The system (37) is the Stokes-Dirac structure.

Proof. We have already proven that the system (37) is a Dirac structure in Proposition 1. On the other hand, the power balance (40) corresponds with the main property of distributed port-Hamiltonian systems described by the Stokes-Dirac structure, and it can be regarded as the higher order version of the structure [12].

\section{Examples}

This section presents two modeling examples.

\subsection{Timoshenko Beam Equation}

The 1-dimensional Timoshenko beam equation

$$
\left\{\begin{array}{l}
A_{\rho} \epsilon_{t t}-G A D_{y}\left(\epsilon_{y}-\theta\right)=0 \\
I_{\rho} \theta_{t t}-E I \theta_{y y}-G A\left(\epsilon_{y}-\theta\right)=0
\end{array}\right.
$$


is derived from the Lagrangian density functional on $\Omega^{2,0}\left(J^{3} Q\right)$

$$
\mathscr{L}=\int_{\mathcal{Y}} \mathcal{L} \mathrm{d} x=\frac{1}{2} \int_{\mathcal{Y}}\left\{A_{\rho} \varepsilon_{t}^{2}+I_{\rho} \theta_{t}^{2}-G A\left(\epsilon_{y}-\theta\right)^{2}-E I \theta_{y}^{2}\right\} \mathrm{d} x,
$$

where $t \in\left[t_{0}, t_{1}\right]$ is the time coordinate, $y \in \mathcal{S}$ is the spatial coordinate along the longitudinal axis, $\epsilon \in \mathbb{R}$ is the shearing, $\theta \in \mathbb{R}$ is the rotation at each point in $y, A_{\rho}$ is the unit mass, $I_{\rho}$ is the moment of inertia, $E I$ is the elastic stiffness, and $G A$ is the shearing stiffness.

Let $m=2, l=2$, and $r=3$. From $\left(x^{0}, x^{1} ; q^{1}, q^{2}\right)=(t, y ; \epsilon(t, y), \theta(t, y))$ and the maximum of higher order degrees $\max |K|=2$ in (44), we derive $q_{K t}^{1}=D_{K t} \epsilon$ for $0 \leq|K t| \leq 3$. By defining $J^{3} Q$ with the local coordinate $q_{K t}^{2}=D_{K t} \theta$, we set $\mathrm{d} x=\mathrm{d} t \wedge \mathrm{d} y$ and $\mathcal{Y}=\left[t_{0}, t_{1}\right] \times \mathcal{S}$.

In (38), from $k=1,0 \leq|K| \leq 1, \quad j=1$, and $a=2$, we obtain

$$
\left\{\begin{array}{l}
\left(f_{1}^{p}, e_{1}^{p}\right)=\left((-1)^{1} D_{t} \tilde{p}_{1}^{t}, \epsilon_{t}\right)=\left(D_{t}\left(A_{\rho} \epsilon_{t}\right), \epsilon_{t}\right), \\
\left(f_{2}^{p}, e_{2}^{p}\right)=\left((-1)^{1} D_{t} \tilde{p}_{2}^{t}, \theta_{t}\right)=\left(D_{t}\left(I_{\rho} \theta_{t}\right), \theta_{t}\right), \\
\left(f_{q}^{2}, e_{q}^{2}\right)=\left(D_{t} \theta, \tilde{p}_{2}\right)=\left(D_{t} \theta, G A\left(\epsilon_{y}-\theta\right)\right), \\
\left(f_{q y}^{1}, e_{q y}^{1}\right)=\left(D_{t} \epsilon_{y}, \frac{1}{w(y)} \tilde{p}_{1}^{\bar{y}}\right)=\left(D_{t} \epsilon_{y},-G A\left(\epsilon_{y}-\theta\right)\right), \\
\left(f_{q y}^{2}, e_{q y}^{2}\right)=\left(D_{t} \theta_{y}, \frac{1}{w(y)} \tilde{p}_{2}^{\bar{y}}\right)=\left(D_{t} \theta_{y},-E I \theta_{y}\right), \\
\left(f_{\partial 1,1}^{1}, e_{\partial 1,1}^{1}\right)=\left(D_{s}^{1-1} q_{t}^{1},-\left(-D_{s}\right)^{1-1} e_{q y}^{1}\right)=\left(\epsilon_{t},-e_{q y}^{1}\right), \\
\left(f_{\partial 1,1}^{2}, e_{\partial 1,1}^{2}\right)=\left(D_{s}^{1-1} q_{t}^{2},-\left(-D_{s}\right)^{1-1} e_{q y}^{2}\right)=\left(\theta_{t},-e_{q y}^{2}\right),
\end{array}\right.
$$

where $D_{s}=D_{y}$ and we have defined

$$
\begin{aligned}
& \tilde{p}_{1}^{t}=\frac{\partial \mathcal{L}}{\partial \varepsilon_{t}}, \quad \tilde{p}_{2}^{t}=\frac{\partial \mathcal{L}}{\partial \theta_{t}}, \\
& \tilde{p}_{2}=\frac{\partial \mathcal{L}}{\partial \theta}, \quad \tilde{p}_{1}^{\bar{y}} \frac{\partial \mathcal{L}}{\partial \varepsilon_{\bar{y}}}, \quad \tilde{p}_{2}^{\bar{y}}=\frac{\partial \mathcal{L}}{\partial \theta_{\bar{y}}} .
\end{aligned}
$$

Hence, we have

$$
\left\{\begin{array}{l}
{\left[\begin{array}{l}
f_{1}^{p} \\
f_{2}^{p} \\
f_{2}^{q} \\
f_{q y}^{1} \\
f_{q y}^{2}
\end{array}\right]=\left[\begin{array}{ccccc}
0 & 0 & D_{y} & 0 & 0 \\
0 & 0 & 0 & -1 & D_{y} \\
D_{y} & 0 & 0 & 0 & 0 \\
0 & 1 & 0 & 0 & 0 \\
0 & D_{y} & 0 & 0 & 0
\end{array}\right]\left[\begin{array}{c}
e_{1}^{p} \\
e_{2}^{p} \\
e_{2}^{q} \\
e_{q y}^{1} \\
e_{q y}^{2}
\end{array}\right],} \\
{\left[\begin{array}{l}
f_{\partial}^{1} \\
f_{\partial}^{2}
\end{array}\right]=\left[\begin{array}{l}
\left.f_{\partial 1,1}^{1}\right|_{\partial \mathcal{S}} \\
\left.f_{\partial 1,1}^{2}\right|_{\partial \mathcal{S}}
\end{array}\right],\left[\begin{array}{l}
e_{\partial}^{1} \\
e_{\partial}^{2}
\end{array}\right]=\left[\begin{array}{l}
\left.e_{\partial 1,1}^{1}\right|_{\partial \mathcal{S}} \\
\left.e_{\partial 1,1}^{2}\right|_{\partial \mathcal{S}}
\end{array}\right],}
\end{array}\right.
$$

where two lines form the first equation in (48) is equivalent to (44), and three lines from the bottom are equalities. Moreover, the system (48) satisfies

$$
\int_{\mathcal{S}}\left(e_{1}^{p} f_{1}^{p}+e_{2}^{p} f_{2}^{p}+e_{q}^{2} f_{q}^{2}+e_{q y}^{1} f_{q y}^{1}+e_{q y}^{2} f_{q y}^{2}\right) \mathrm{d} x^{s}+\int_{\partial \mathcal{S}}\left(e_{\partial}^{1} f_{\partial}^{1}+e_{\partial}^{2} f_{\partial}^{2}\right) \boldsymbol{i}_{\partial x^{s}} \mathrm{~d} x^{s}=0,
$$

where note that Stokes theorem cannot be applied to $\left(e_{q}^{2}, f_{q}^{2}\right)$; therefore the corresponding boundary term has not been defined. 


\subsection{Potential Boussinesq Equation}

The 1-dimensional potential Boussinesq equation ([27], p. 237) that expresses shallow water waves

$$
q_{y y t t}+\frac{1}{2} D_{y}^{2}\left(q_{y y}^{2}\right)+q_{y y y y y}=0
$$

is derived from the Lagrangian density functional defined on $\Omega^{2,0}\left(J^{7} Q\right)$

$$
\mathscr{L}=\int_{\mathcal{y}}\left(\frac{1}{2} q_{y t}^{2}+\frac{1}{6} q_{y y}^{3}-\frac{1}{2} q_{y y y}^{2}\right) \mathrm{d} x,
$$

where $t \in\left[t_{0}, t_{1}\right]$ is the time coordinate, $y \in \mathcal{S}$ is the spatial coordinate along the water surface, and $q \in \mathbb{R}$ is the height of the wave.

Let $m=2, \quad l=1$, and $r=7$. We have defined $J^{7} Q$ with the local coordinate $q_{K t}^{1}=D_{K t} q$ for $0 \leq|K t| \leq 7, \mathrm{~d} x=\mathrm{d} t \wedge \mathrm{d} y$, and $\mathcal{Y}=\left[t_{0}, t_{1}\right] \times \mathcal{S}$ by $\left(x^{0}, x^{1} ; q^{1}\right)=(t, y ; q(t, y))$ and the maximum order $\max |K|=6$ in (50).

By substituting $k=3,0 \leq|K| \leq 3,1 \leq j \leq 3$, and $a=1$ to (38), we get

$$
\left\{\begin{array}{l}
\left(f_{1}^{p}, e_{1}^{p}\right)=\left((-1)^{2} D_{y t} \tilde{p}_{1}^{y t}, q_{t}\right)=\left(D_{y t} q_{y t}, q_{t}\right), \\
\left(f_{q y y}^{1}, e_{q y y}^{1}\right)=\left(D_{t} q_{y y}, \frac{1}{w(y y)} \tilde{p}_{1}^{\overline{y y}}\right)=\left(D_{t} q_{y y}, \frac{1}{2} q_{y y}^{2}\right) \\
\left(f_{q y y y}^{1}, e_{q y y y}^{1}\right)=\left(D_{t} q_{y y y}, \frac{1}{w(y y y)} \tilde{p}_{1}^{\overline{y y y}}\right)=\left(D_{t} q_{y y y},-q_{y y y}\right), \\
\left(f_{\partial 2,1}^{1}, e_{\partial 2,1}^{1}\right)=\left(D_{s}^{2-1} q_{t}^{1},-\left(-D_{s}\right)^{1-1} e_{q y y}^{1}\right)=\left(D_{y} q_{t},-e_{q y y}^{1}\right), \\
\left(f_{\partial 2,2}^{1}, e_{\partial 2,2}^{1}\right)=\left(D_{s}^{2-2} q_{t}^{1},-\left(-D_{s}\right)^{2-1} e_{q y y}^{1}\right)=\left(q_{t}, D_{y} e_{q y y}^{1}\right), \\
\left(f_{\partial 3,1}^{1}, e_{\partial 3,1}^{1}\right)=\left(D_{s}^{3-1} q_{t}^{1},-\left(-D_{s}\right)^{1-1} e_{q y y y}^{1}\right)=\left(D_{y}^{2} q_{t},-e_{q y y y}^{1}\right), \\
\left(f_{\partial 3,2}^{1}, e_{\partial 3,2}^{1}\right)=\left(D_{s}^{3-2} q_{t}^{1},-\left(-D_{s}\right)^{2-1} e_{q y y y}^{1}\right)=\left(D_{y} q_{t}, D_{y} e_{q y y y}^{1}\right), \\
\left(f_{\partial 3,3}^{1}, e_{\partial 3,3}^{1}\right)=\left(D_{s}^{3-3} q_{t}^{1},-\left(-D_{s}\right)^{3-1} e_{q y y y}^{1}\right)=\left(q_{t},-D_{y}^{2} e_{q y y y}^{1}\right),
\end{array}\right.
$$

where $D_{s}=D_{y}$ and we have defined

$$
\tilde{p}_{1}^{y t}=\frac{\partial \mathcal{L}}{\partial q_{y t}}, \tilde{p}_{1}^{\overline{y y}}=\frac{\partial \mathcal{L}}{\partial q_{\overline{y y}}}, \tilde{p}_{1}^{\overline{y y y}}=\frac{\partial \mathcal{L}}{\partial q_{\overline{y y y}}}
$$

Hence, the following system representation is given:

$$
\left\{\begin{array}{l}
{\left[\begin{array}{c}
f_{p}^{1} \\
f_{q y y}^{1} \\
f_{q y y y}^{1}
\end{array}\right]=\left[\begin{array}{ccc}
0 & -D_{y}^{2} & D_{y}^{3} \\
D_{y}^{2} & 0 & 0 \\
D_{y}^{3} & 0 & 0
\end{array}\right]\left[\begin{array}{c}
e_{p}^{1} \\
e_{q y y}^{1} \\
e_{q y y y}^{1}
\end{array}\right],} \\
f_{\partial}^{1}=\left[\begin{array}{l}
\left.f_{\partial 2,1}^{1}\right|_{\partial \mathcal{S}} \\
\left.f_{\partial 2,2}^{1}\right|_{\partial \mathcal{S}} \\
\left.f_{\partial 3,1}^{1}\right|_{\partial \mathcal{S}} \\
\left.f_{\partial 3,2}^{1}\right|_{\partial \mathcal{S}} \\
\left.f_{\partial 3,3}^{1}\right|_{\partial \mathcal{S}}
\end{array}\right], e_{\partial}^{1}=\left[\begin{array}{l}
\left.e_{\partial 2,1}^{1}\right|_{\partial \mathcal{S}} \\
\left.e_{\partial 2,2}^{1}\right|_{\partial \mathcal{S}} \\
\left.e_{\partial 3,1}^{1}\right|_{\partial \mathcal{S}} \\
\left.e_{\partial 3,2}^{1}\right|_{\partial \mathcal{S}} \\
\left.e_{\partial 3,3}^{1}\right|_{\partial \mathcal{S}}
\end{array}\right],
\end{array}\right.
$$


where the first line of the first equation in (54) is equivalent to (50), and two lines from the bottom are equalities. Moreover, the system (54) satisfies

$$
\int_{\mathcal{S}}\left(e_{1}^{p} f_{1}^{p}+e_{q y y}^{1} f_{q y y}^{1}+e_{q y y y}^{1} f_{q y y y}^{1}\right) \mathrm{d} x^{s}+\int_{\partial \mathcal{S}}\left\{\left(e_{\partial}^{1}\right)^{\mathrm{T}} f_{\partial}^{1}\right\} \boldsymbol{i}_{\partial x^{s}} \mathrm{~d} x^{s}=0 .
$$

\section{Conclusions}

This paper derived the higher order field port Hamiltonian system with boundary energy flows from systems of higher order partial differential equations that are determined by variational problems in terms of the multisymplectic instantaneous formalism. By defining the symplectic structure induced from distributions and the Stokes variational differential including the integration by parts operators, we clarified the implicit Hamiltonian representation of the systems of higher order partial differential equations, and its local expression corresponds to the distributed port-Hamiltonian system.

In this paper, we assumed that Lagrangians are first order with respect to time, but possibly higher order with respect to spatial variables for simplification. This assumption can be generalized. On the other hand, the formal representation including time derivatives up to first order corresponds to the distributed port-Hamiltonian systems.

\section{Acknowledgements}

The author thanks Professor Bernhard Maschke for fruitful discussions on this study. This work was supported by JSPS Grants-in-Aid for Scientific Research (C) No. 26420415, and JSPS Grants-in-Aid for Challenging Exploratory Research No. 26630197.

\section{References}

[1] Abraham, R. and Marsden, J. (2008) Foundations of Mechanics. 2nd Edition, AMS Chelsea Pub., Chelsea.

[2] van der Schaft, A.J. (2000) $\mathrm{L}_{2}$-Gain and Passivity Techniques in Nonlinear Control. 2nd Revised and Enlarged Edition, Springer-Verlag, London. http://dx.doi.org/10.1007/978-1-4471-0507-7

[3] Duindam, V., Macchelli, A., Stramigioli, S. and Bruyninckx, H., Eds. (2009) Modeling and Control of Complex Physical Systems—The Port-Hamiltonian Approach. Springer, Berlin. http://dx.doi.org/10.1007/978-3-642-03196-0

[4] Karnopp, D.C., Margolis, D.L. and Rosenberg, R.C. (2006) System Dynamics, Modeling and Simulation of Mechatronic Systems. 4th Edition, Wiley, Hoboken.

[5] Gotay, M.J. (1991) A Multisymplectic Framework for Classical Field Theory and the Calculus of Variations: I. Covariant Hamiltonian Formalism. In: Francaviglia, M., Ed., Mechanics, Analysis and Geometry: 200 Years after Lagrange, Elsevier Science Pub. B.V., Amsterdam, 203-235. http://dx.doi.org/10.1016/B978-0-444-88958-4.50012-4

[6] Gotay, M.J. (1991) A Multisymplectic Framework for Classical Field Theory and the Calculus of Variations II: Space + Time Decomposition. Differential Geometry and Its Applications, 1, 375-390. http://dx.doi.org/10.1016/0926-2245(91)90014-Z

[7] van der Schaft, A.J. and Maschke, B.M. (2002) Hamiltonian Formulation of Distributed-Parameter Systems with Boundary Energy Flow. Journal of Geometry and Physics, 42, 166-194. http://dx.doi.org/10.1016/S0393-0440(01)00083-3

[8] Macchelli, A. (2014) Passivity-Based Control of Implicit Port-Hamiltonian Systems. SIAM Journal on Control and Optimization, 52, 2422-2448. http://dx.doi.org/10.1137/130918228

[9] Nishida, G. and Yamakita, M. (2005) Formal Distributed Port-Hamiltonian Representation of Field Equations. Proceedings of the 44th IEEE Conference on Decision and Control, Seville, 12-15 December 2005, 6009-6015. http://dx.doi.org/10.1109/CDC.2005.1583123

[10] Nishida, G. and Maschke, B. (2012) Implicit Representation for Passivity-Based Boundary Controls. Proceedings of the 4th IFAC Workshop on Lagrangian and Hamiltonian Methods for Non-Linear Control, Bertinoro, 29-31 August 2012, 200-207.

[11] Schöberl, M. and Siuka, A. (2014) Jet Bundle Formulation of Infinite-Dimensional Port-Hamiltonian Systems Using Differential Operators. Automatica, 50, 607-613. http://dx.doi.org/10.1016/j.automatica.2013.11.035

[12] Nishida, G. and Yamakita, M. (2004) A Higher Order Stokes-Dirac Structure for Distributed-Parameter Port-Hamiltonian Systems. Proceedings of the 2004 American Control Conference, Boston, 30 June-2 July 2004, 5004-5009. 
[13] Le Gorrec, Y., Zwart, H. and Maschke, B. (2005) Dirac Structures and Boundary Control Systems Associated with Skew-Symmetric Differential Operators. SIAM Journal on Control and Optimization, 44, 1864-1892. http://dx.doi.org/10.1137/040611677

[14] van der Schaft, A.J. (1998) Implicit Hamiltonian Systems with Symmetry. Reports on Mathematical Physics, 41, 203221. http://dx.doi.org/10.1016/S0034-4877(98)80176-6

[15] Dalsmo, M. and van der Schaft, A. (1998) On Representations and Integrability of Mathematical Structures in EnergyConserving Physical Systems. SIAM Journal on Control and Optimization, 37, 54-91. http://dx.doi.org/10.1137/S0363012996312039

[16] Yoshimura, H. and Marsden, J.E. (2006) Dirac Structures in Lagrangian Mechanics Part I: Implicit Lagrangian Systems. Journal of Geometry and Physics, 57, 133-156. http://dx.doi.org/10.1016/j.geomphys.2006.02.009

[17] Vankerschaver, J., Yoshimura, H. and Leok, M. (2012) The Hamilton-Pontryagin Principle and Multi-Dirac Structures for Classical Field Theories. Journal of Mathematical Physics, 53, Article ID: 072903. http://dx.doi.org/10.1063/1.4731481

[18] Courant, T. (1990) Dirac Manifolds. Transactions of the American Mathematical Society, 319, 631-661. http://dx.doi.org/10.1090/S0002-9947-1990-0998124-1

[19] Dorfman, I. (1993) Dirac Structures and Integrability of Nonlinear Evolution Equations. John Wiley, Chichester.

[20] Nishida, G., Maschke, B. and Ikeura, R. (2015) Boundary Integrability of Multiple Stokes-Dirac Structures. SIAM Journal Control and Optimization, 53, 800-815. http://dx.doi.org/10.1137/110856058

[21] Nishida, G., Yamakita, M. and Luo, Z. (2007) Virtual Lagrangian Construction Method for Infinite Dimensional Systems with Homotopy Operators. In: Allgüwer, F., Fleming, P., Kokotovic, P., Kurzhanski, A.B., Kwakernaak, H., Rantzer, A., et al., Eds., Lagrangian and Hamiltonian Methods for Nonlinear Control 2006, Lecture Notes in Control and Information Sciences, Vol. 366, Springer, Berlin, 75-86. http://dx.doi.org/10.1007/978-3-540-73890-9_5

[22] Olver, P.J. (1993) Applications of Lie Groups to Differential Equations. 2nd Edition, Springer-Verlag, New York. http://dx.doi.org/10.1007/978-1-4612-4350-2

[23] Anderson, I.M. (1992) Introduction to the Variational Bicomplex. Contemporary Mathematics, 132, 51-73. http://dx.doi.org/10.1090/conm/132/1188434

[24] Saunders, D.J. (1989) The Geometry of Jet Bundles. Cambridge University Press, Cambridge. http://dx.doi.org/10.1017/CBO9780511526411

[25] Giachetta, G., Mangiarotti, L. and Sardanashvily, G. (1997) New Lagrangian and Hamiltonian Methods in Field Theory. World Scientific, Singapore. http://dx.doi.org/10.1142/2199

[26] Tulczyjew, W.M. (1977) The Legendre Transformation. Annales de l'Institut Henri Poincaré (A) Physique Théorique, 27, 101-114.

[27] Olver, P.J. (1995) Equivalence, Invariants, and Symmetry. Cambridge University Press, Cambridge. http://dx.doi.org/10.1017/CBO9780511609565

\section{Appendix}

\section{A. Passivity}

Consider the following pairing between $L_{e}^{q}(U)$ and $L_{e}^{q}\left(U^{*}\right)$ :

$$
\langle y, u\rangle_{T}:=\int_{0}^{T}\langle y(t), u(t)\rangle \mathrm{d} t
$$

for $u \in L_{e}^{q}(U)$ and $y \in L_{e}^{q}\left(U^{*}\right)$, where $\langle$,$\rangle is the duality product, U$ is a finite dimensional linear space, $U^{*}$ is its dual space, and we have defined the extended $L_{e}^{q}$ space that is the set of all measurable functions in $L^{q}$ truncated to a finite time interval. Note that the duality product $\langle y(t), u(t)\rangle$ corresponds to power.

Definition 10. Let $G: L_{e}^{q}(U) \rightarrow L_{e}^{q}\left(U^{*}\right)$. Then $G$ is passive if there exists a constant $H$ such that 


$$
\langle G u, u\rangle_{T} \geq-H, \quad \forall u \in L^{q}(U), \quad \forall T \geq 0,
$$

where the left-side of (57) is assumed to be well-defined.

Hence, $G$ is passive if and only if a finite amount of energy can be extracted from the system defined by $G$.

Corollary 3. For a point $t$ in the time axis,

$$
\langle G u(t), u(t)\rangle \geq-\frac{\mathrm{d} H}{\mathrm{~d} t}, \quad \forall u \in L^{q}(U) .
$$

\section{B. Differential Forms on Bundles}

A differential $(i, j)$-form $g \eta \wedge \psi$ defined on the $r$-th order jet bundle $J^{r} Q$ are defined by a horizontal $i$-form $\psi=\mathrm{d} x^{1} \wedge \cdots \wedge \mathrm{d} x^{i} \in \Omega_{h}^{i}\left(J^{r} Q\right)$ and a vertical $j$-form $\eta=\mathrm{d} q_{I_{1}}^{a_{1}} \wedge \cdots \wedge \mathrm{d} q_{I_{j}}^{a_{j}} \in \Omega_{v}^{j}\left(J^{r} Q\right)$, where $\Omega^{j}\left(J^{r} Q\right)$ is the space of differential $j$-forms on $J^{r} Q, g=g\left(x^{i}, q_{I}^{a}\right)$ is a smooth function defined on $J^{r} Q$, and $a_{k}$ and $I_{k}$ are different combination selected from $a$ and $I$ for $1 \leq k \leq j$.

Let $\Omega^{i, j}\left(J^{r} Q\right)$ be the space of differential $(i, j)$-forms defined on $J^{r} Q$. (i,j) -forms such that $n=i+j$ are called $n$-forms, and their space is denoted by $\Omega^{n}\left(J^{r} Q\right)$.

The exterior differential operator $\mathrm{d}_{=} \mathrm{d}_{v}+\mathrm{d}_{h}$ for $(i, j)$-forms is defined by the vertical differential operator

$$
\mathrm{d}_{v}: \Omega^{i, j}\left(J^{r} Q\right) \rightarrow \Omega^{i, j+1}\left(J^{r} Q\right) ; g \eta \wedge \psi \mapsto \sum_{a, I} \frac{\partial g}{\partial q_{I}^{a}} \mathrm{~d} q_{I}^{a} \wedge \eta \wedge \psi
$$

and the horizontal differential operator

$$
\mathrm{d}_{h}: \Omega^{i, j}\left(J^{r} Q\right) \rightarrow \Omega^{i+1, j}\left(J^{r} Q\right) ; g \eta \wedge \psi \mapsto \sum_{k=1}^{m} D_{k} g \eta \wedge \mathrm{d} x^{k} \wedge \psi,
$$

where the total differential operator with respect to $x^{i}$ is defined by

$$
D_{i}=\frac{\partial}{\partial x^{i}}+\sum_{a=1 \mid[I \mid=0}^{l} \sum_{I i}^{\infty} \frac{\partial}{\partial q_{I}^{a}}
$$

that is equivalent to partial differential, and $D_{i} \mathrm{~d} q_{I}=\mathrm{d}\left(D_{i} q_{I}\right)=\mathrm{d} q_{I, i}$. Note that $q_{I, i}=\partial q_{I} / \partial x^{i}$, and $q_{I i}=(w(I) / w(I i)) q_{I, i}$, where $w(I)$ is the weight of the index $I$ [22] [24], and $q_{I}^{a}=(1 / w(I)) q_{\bar{I}}^{a}$ for the index $\bar{I}$ generated by the repeated permutation of the combination in $I$.

\section{Euler-Lagrange Equations}

An Euler-Lagrange equation is determined by the stationary condition $\mathrm{d} \mathcal{L} \mathrm{d} x=0$ of the variational derivative $\mathrm{d} \mathcal{L}$ of a Lagrange density $\mathcal{L}=\mathcal{L} \mathrm{d} x \in \Omega^{m, 0}\left(J^{r} Q\right)$. If variables on boundaries are zero, the local expression of Euler-Lagrange equations is given by the stationary condition of

$$
\mathrm{d}_{v} \mathcal{L} \mathrm{d} x=\frac{\partial \mathcal{L}}{\partial q_{I}^{a}} \mathrm{~d} q_{I}^{a} \wedge \mathrm{d} x=(-1)^{|I|} D_{I} \frac{\partial \mathcal{L}}{\partial q_{I}^{a}} \mathrm{~d} q^{a} \wedge \mathrm{d} x,
$$

where $\mathrm{d} x=\mathrm{d} x^{0} \wedge \cdots \wedge \mathrm{d} x^{m}$ is the $(m+1)$-volume form, $D_{I}$ is the total differential operator $D_{i}$ with respect to all index in $I$, integration by parts is used in the second equality, and the term $\sum_{i=0}^{m} D_{i}(\cdot)$ is eliminated by Stokes theorem under the assumption of the zero boundary condition.

\section{Multisymplectic Covariant Formalism}

The Hamiltonian representation of lumped parameter systems are determined by the symplectic 2-form $\omega=-\mathrm{d} \theta$ on cotangent bundle $T^{*} Q$, where $\theta=p \mathrm{~d} q$ is the canonical 1-form. Then, for a given Hamiltonian $H: T^{*} Q \rightarrow \mathbb{R}$, Hamiltonian vector field $\mu$ is defined by $\boldsymbol{i}_{\mu} \omega=\mathrm{d} H \quad$ ([1], p. 187), where $(q, p) \in T^{*} Q, \quad \boldsymbol{i}$ is 
the interior product.

The (covariant) Hamiltonian representation of field equations are determined by the multisymplectic $(m+2)$ form $\Omega=-\mathrm{d} \Theta$ on the multisymplectic manifold $Z^{r-1}$ ([5], p. 211), where $\Theta$ is the canonical $(m+1)$-form. Then, $Z^{r-1}$ is defined as the subbundle of $\wedge^{m+1}\left(J^{r-1} Q\right)$ over $J^{r-1} Q$ defining $(m+1)$-forms

$$
Z^{r-1}=\left\{z \in \wedge^{m+1}\left(J^{r-1} Q\right) \mid \boldsymbol{i}_{\xi} \boldsymbol{i}_{v} z=0, \forall v, \xi \in V J^{r-1} Q\right\},
$$

where the space $\Omega^{m+1}\left(J^{r-1} Q\right)$ of $(m+1)$-forms over $J^{r-1} Q$ is defined by the space of all sections of $(m+1)$-th degree exterior power cotangent $(r-1)$-th jet bundle [5], and the vertical tangent bundle $V \mathcal{Y}$ has been defined as a vector subbundle $T \mathcal{Y} \supset V \mathcal{Y}=\operatorname{Ker} T \pi$ determined by the tangent map $T \pi: T \mathcal{Y} \rightarrow T X$ for a bundle $\pi: \mathcal{Y} \rightarrow X$. Note that the local coordinates $\left(x^{i}, \dot{X}^{i}\right)$ of the tangent bundle $T X$ when those of the manifold $X$ are $\left(x^{i}\right)$, and the local coordinates of $T Y$ are $\left(x^{i}, y^{a}, \dot{x}^{i}, \dot{y}^{a}\right)$ when those of the bundle $\pi: Y \rightarrow X$ are $\left(x^{i}, y^{a}\right)$, where those of $V Y$ are $\left(x^{i}, y^{a}, \dot{y}^{a}\right)$. Let $\left(x^{i} ; q_{K}^{a}, p_{a}^{K i}, p\right)$ for $0 \leq|K| \leq r-1$ be the local coordinates of $Z^{r-1}$. Any $z \in Z^{r-1}$ in (63) can be locally written as

$$
z=p \mathrm{~d} x+\sum_{|K|=0}^{r-1} p_{a}^{K i} \mathrm{~d} q_{K}^{a} \wedge \boldsymbol{i}_{\partial x^{i}} \mathrm{~d} x,
$$

where we assume that the multi index Ki satisfies $|K i|=|K|+1$. Then, $\Theta=z$ is defined by using (64).

On the other hand, the covariant Lagrangian system can be defined as $-\mathrm{d}_{\mathcal{L}}$ on $J^{2 r-1} Q$ by the Cartan form $\Theta_{\mathcal{L}} \in \Omega^{m+1}\left(J^{2 r-1} Q\right)$, where $\sigma_{\mathcal{L}}^{*} \Theta=\Theta_{\mathcal{L}}$, and $\sigma_{\mathcal{L}}: J^{2 r-1} Q \rightarrow Z^{r-1}$ is the covariant Legendre transformation on $J^{r-1} Q$

$$
\begin{cases}p_{a}^{K}=\frac{\partial \mathcal{L}}{\partial q_{K}^{a}}+c_{a}^{K} & \text { if }|K|=r, \\ p_{a}^{K}=\frac{\partial \mathcal{L}}{\partial q_{K}^{a}}-D_{i} p_{a}^{K i}+c_{a}^{K} & \text { if } 1 \leq|K|<r, \\ c_{a}^{K}=0 & \text { if }|K|=1, \\ p=\mathcal{L}-\sum_{|K| 0}^{r-1} p_{a}^{K i} q_{K i}^{a}, & \end{cases}
$$

where $\sigma_{\mathcal{L}}^{*}$ is the pull-back, and $\left(x^{i} ; q_{I}^{a}\right)$ are the local coordinates of $J^{r} Q$ for $0 \leq|I| \leq r$. The functions $c_{a}^{K i}$ and $c_{a}^{K}$ in (65) give arbitrariness in the global expression of $\Theta_{\mathcal{L}}$; therefore, this is not used in the local expression, i.e., $\quad c_{a}^{K i}=c_{a}^{K}=0$.

In the above, the covariant Lagrangian system determined by the variational problem of the $r$-th order Lagrangian density $\mathcal{L} \mathrm{d} x \in \Omega^{m, 0}\left(J^{r} Q\right)$ on $J^{r} Q$ is defined by the Cartan form $\Theta_{\mathcal{L}} \in \Omega^{m+1}\left(J^{2 r-1} Q\right)$ on $J^{2 r-1} Q$ ([5], p. 210). Note that the covariant Hamiltonian $p$ determines the affine structure of $Z^{r-1}$ that is the essential of the symplectic structure.

\section{E. Multisymplectic Instantaneous Formalism}

The instantaneous formalism is the covariant representation with time-spatial splitting. The time-spatial splitting is equivalent to choosing an infinitesimal supersurface parametrized by time in the configuration space $Q$. Bundles with time-spatial splitting consist of the Cauchy surface $\Sigma \subset X$ and time-spatial vector fields $\zeta$ on $Q$. Then, $\zeta$ means the direction of the time evolution of the system, and $\zeta$ on $\left.Q\right|_{\Sigma}$ transversally intersects to $\left.Q\right|_{\Sigma}$ everywhere, where $\left.Q\right|_{\Sigma}$ means the restricted $Q$ to $\Sigma$.

The instantaneous representation is defined on the space $\Gamma\left(\left.J^{2 r-1} Q\right|_{\Sigma}\right)$ that consists of all sections $\gamma$ of 
$J^{2 r-1} Q$ restricted to $\Sigma$. For a given global section $\gamma \in \Gamma\left(\left.J^{2 r-1} Q\right|_{\Sigma}\right)$, the local coordinates of $\Gamma\left(\left.J^{2 r-1} Q\right|_{\Sigma}\right)$ is given as $\gamma_{J}^{a}=q_{J}^{a} \circ \gamma$ ([6], p. 379), where $q_{J}^{a}$ is the local coordinates of $J^{2 r-1} Q$ for $0 \leq|J| \leq 2 r-1$. Then, the subbundle $\left.j^{2 r-1} Q\right|_{\Sigma}$ of $\Gamma\left(\left.J^{2 r-1} Q\right|_{\Sigma}\right)$ that can be identified with the tangent bundle $\left.T^{2 r-1} Q\right|_{\Sigma}$ by choosing the direction of the time evolution of $\zeta$, where the local coordinates of $\left.j^{2 r-1} Q\right|_{\Sigma}$ are obtained from $\gamma_{U}^{a}$ by using the multi index $U$ with respect to time for $0 \leq|U| \leq 2 r-1$. Hence, by restricting the system to $\left.j^{2 r-1} Q\right|_{\Sigma}$, the spatial derivatives in $\gamma_{J}^{a}$ are eliminated.

$\left.T^{2 r-1} Q\right|_{\Sigma}$ and $T^{*}\left(\left.T^{r-1} Q\right|_{\Sigma}\right)$ are the vector bundle over $\left.T^{r-1} Q\right|_{\Sigma}$ with $2 r$ numbers of local coordinates, respectively, $\left(\gamma_{L}^{a}\right)$ for $0 \leq|J| \leq 2 r-1$ and $\left(\gamma_{K}^{a}, \pi_{a}^{K}\right)$ for $0 \leq|K| \leq r-1$. On the dual bundle of $\left.T^{2 r-1} Q\right|_{\Sigma}$, i.e., $T^{*}\left(\left.T^{r-1} Q\right|_{\Sigma}\right)$, the instantaneous Hamiltonian systems are derived from the canonical form

$$
\vartheta_{\Sigma}=\int_{\Sigma}\left(\sum_{|L|=0}^{r-1} \pi_{a}^{L} \mathrm{~d} q_{L}^{a}\right) \mathrm{d} x^{s},
$$

where the instantaneous momentum

$$
\pi_{a}^{L}=\sum_{|K|=0}^{r-|L|-1}(-1)^{|K|} w(L, K) D_{K} p_{a}^{K L t}
$$

is calculated by integration by parts, and the weight

$$
w(L, K)=(|L|+|K|) ! /(|L| !|K| !)
$$

based on the combination have been defined.

Instantaneous Lagrange systems are determined on $\left.T^{2 r-1} Q\right|_{\Sigma}$ by $\vartheta_{\mathcal{L}, \Sigma}=\sigma_{\mathcal{L}, \Sigma}^{*} \vartheta_{\Sigma} \quad$ [6, pp. 382], where the instantaneous Legendre transformation

$$
\sigma_{\mathcal{L}, \Sigma}:\left.T^{2 r-1} Q\right|_{\Sigma} \rightarrow T^{*}\left(\left.T^{r-1} Q\right|_{\Sigma}\right)
$$

is given by $\gamma_{U}^{a} \mapsto\left(\gamma_{L}^{a}, \pi_{a}^{L}\right)$ for $0 \leq|U| \leq 2 r-1$ and $0 \leq|L| \leq r-1$ that are multi indexes with respect to time. The image in $T^{*}\left(\left.T^{r-1} Q\right|_{\Sigma}\right)$ of the bundle map $\sigma_{\mathcal{L}, \Sigma}$ is the instantaneous primal constraint set $P_{\Sigma}^{r-1}$. The instantaneous Hamiltonian $\mathscr{K}_{\Sigma}: P_{\Sigma}^{r-1} \rightarrow \mathbb{R}$ on $P_{\Sigma}^{r-1}$ is defined as follows ([6], p. 384):

$$
\mathscr{K}_{\Sigma}=\int_{\Sigma}\left(\sum_{|L|=0}^{r-1} \pi_{a}^{L} q_{L t}^{a}-\mathcal{L}\right) \mathrm{d} x^{s} .
$$

Because $P_{\Sigma}^{r-1}$ possesses the (pre-)symplectic structure $\omega_{\Sigma}$ of $T^{*}\left(\left.T^{r-1} Q\right|_{\Sigma}\right)$, instantaneous Hamiltonian systems on $P_{\Sigma}^{r-1}$ are given by evolutional vector fields $v$ such that $\boldsymbol{i}_{v} \omega_{\Sigma}=\mathrm{d} \mathscr{C} \Sigma$. 